\title{
Reversible Deactivation of Motor Cortex Reveals Functional Connectivity with Posterior Parietal Cortex in the Prosimian Galago (Otolemur garnettii)
}

\author{
Dylan F. Cooke, ${ }^{1}$ Iwona Stepniewska, ${ }^{2}$ Daniel J. Miller, ${ }^{2}$ Jon H. Kaas, ${ }^{2}$ and Leah Krubitzer ${ }^{1}$ \\ ${ }^{1}$ Center for Neuroscience and Department of Psychology, University of California, Davis, California 95618, and 2Department of Psychology, Vanderbilt \\ University, Nashville, Tennessee 37203
}

\begin{abstract}
We examined the functional macrocircuitry of frontoparietal networks in the neocortex of prosimian primates (Otolemur garnettii) using a microfluidic thermal regulator to reversibly deactivate selected regions of motor cortex (M1). During deactivation of either forelimb or mouth/face movement domains within M1, we used long-train intracortical microstimulation techniques to evoke movements from the rostral division of posterior parietal cortex (PPCr). We found that deactivation of M1 movement domains in most instances abolished movements evoked in PPCr. The most common effect of deactivating M1 was to abolish evoked movements in a homotopic domain in PPCr. For example, deactivating M1 forelimb lift domains resulted in loss of evoked movement in forelimb domains in PPCr. However, at some sites, we also observed heterotopic effects; deactivating a specific domain in M1 (e.g., forelimb lift) resulted in loss of evoked movement in a different movement domain in PPCr (e.g., hand-to-mouth or eye-blink). At most sites examined in PPCr, rewarming M1 resulted in a reestablishment of the baseline movement at the same amplitude as that observed before cooling. However, at some sites, reactivation did not result in a return to baseline movement or to the full amplitude of the baseline movement. We discuss our findings in the context of frontoparietal circuits and how they may subserve a repertoire of ecologically relevant behaviors.
\end{abstract}

Key words: cooling inactivation; lesion; muscimol; network; primate; reaching

Significance Statement

The posterior parietal cortex (PPC) of primates integrates sensory information used to guide movements. Different modules within PPC and motor cortex (M1) appear to control various motor behaviors (e.g., reaching, defense, and feeding). How these modules work together may vary across species and may explain differences in dexterity and even the capacity for tool use. We investigated the functional connectivity of these modules in galagos, a prosimian primate with relatively simple frontoparietal circuitry. By deactivating a reaching module in M1, we interfered with the function of similar PPC modules and occasionally unrelated PPC modules as well (e.g., eye blink). This circuitry in galagos, therefore, is more complex than in nonprimates, indicating that it has been altered with the expansion of primate PPC.

\section{Introduction}

One of the functions of posterior parietal cortex (PPC) is to construct an internal model of the physical self: our body's

Received April 15, 2015; revised Aug. 28, 2015; accepted Sept. 13, 2015.

Author contributions: D.F.C., I.S., J.H.K., and L.K. designed research; D.F.C., I.S., D.J.M., and L.K. performed research; D.F.C. and L.K. analyzed data; D.F.C. and L.K. wrote the paper.

This work was supported by the National Institute of Neurological Disorders and Stroke-National Institutes of Health (Grant NS035103 to L.K., Grant NS16446 to J.H.K., Grant 5 T32 EY007135 to D.J.M., and National Eye Institute Core Grant P30EY012576). We thank Arnold Chen and Adam Goldring for technical assistance.

The authors declare no competing financial interests.

Correspondence should be addressed to Leah Krubitzer, Center for Neuroscience and Department of Psychology, University of California, 1544 Newton Ct., Davis, CA 95618. E-mail: lakrubitzer@ucdavis.edu.

DOI:10.1523/JNEUROSCI.1468-15.2015

Copyright $\odot 2015$ the authors $\quad 0270-6474 / 15 / 3514406-17 \$ 15.00 / 0$ configuration, the boundary between our body and external objects, and the temporary expansion of that self as we wield a tool that extends our reach and capabilities. However, comprehension of where the self is and even the ability to manipulate objects and use them as tools did not evolve de novo in humans, but rather emerged from simpler networks present in early primates, some 80 million years ago.

Over a decade ago, it became clear that traditional views of mammalian motor cortex and PPC did not capture their full complexity. Rather than representing individual muscles or small muscle groups in a topographic fashion (Asanuma and Rosen, 1972; McGuinness et al., 1980), multiple lines of evidence indicated that motor cortex was organized as a mosaic, with body parts represented multiple times in a gross topographic order 
(Gould et al., 1986). Subsequently, an alternative scheme has emerged in macaque monkeys with motor cortex composed of ethologically relevant, complex movement domains overlaid upon a simple, grossly topographic map of the body (Graziano et al., 2002; Cooke and Graziano, 2004; Graziano et al., 2005; Maranesi et al., 2012).

Recently, such domains have been demonstrated in the motor cortex (M1) of squirrel monkeys, owl monkeys, galagos, and rats, indicating that this organization is a shared feature of primate motor cortex (Gharbawie et al., 2011a; Stepniewska et al., 2014), and likely mammals in general (Brown and Teskey, 2014). Further, studies in New World monkeys (Kaas et al., 2011, 2013 for review) and galagos (Stepniewska et al., 2005, 2009a; Stepniewska et al., 2011) demonstrate that, like M1, PPC contains domains of reach, grasp, defense, hand-to-mouth, and other ethologically relevant movements. Somewhat similar (although dispersed) domains have also been described in PPC of macaque monkeys (for review, see Thier and Andersen, 1996; Cooke et al., 2003; Rozzi et al., 2008; Gharbawie et al., 2011a; Kaas et al., 2013). PPC in macaque monkeys has largely been explored using electrophysiological techniques in awake animals. In these studies and in imaging studies in humans, organizational schemes have been described as effector-specific areas with head-centered or eye-centered frames of reference (Colby, 1998; Culham et al., 2006; Hinkley et al., 2009; Andersen et al., 2014; Hwang et al., 2014). Although there are differences between these effector-specific pathways in macaques and the ethologically relevant domains described with intracortical microstimulation (ICMS) in PPC in New World monkeys and galagos, they are similar in that the PPC areas associated with these effector pathways are often action oriented. For example, head-centered representations can guide reaching with the mouth and arm-centered frames of reference can guide reaching, grasping, and preshaping of the hand.

The question, of course, is how action domains in PPC functionally interact with movement domains in M1 to generate and select a movement plan and execute appropriate behavior. To investigate this, we implanted microfluidic cooling devices to reversibly deactivate specific movement domains in M1 of anesthetized galagos. These techniques have been used successfully in brains ranging in size from rats (Brown and Teskey, 2014) to macaque monkeys (Ponce et al., 2011; Goldring et al., 2014). This allowed us to determine how rapidly the network responds to perturbations in motor cortex and the network dynamics when function is rapidly returned (as opposed to slowly reversing chemical deactivations using muscimol, e.g., Hikosaka and Wurtz, 1985; Stepniewska et al., 2014; or plasticity after permanent lesions, e.g., Padberg et al., 2010). By deactivating M1 and examining the effects on evoked movements in PPC, we can determine the extent to which divergent connections from PPC to M1 affect motor output in the relatively simple primate network of the prosimian galago.

\section{Materials and Methods}

Subjects. Four adult galagos (Otolemur garnettii; 2 female and 2 male) ranging in age from 1.2 to 10.6 years and ranging in weight from 1.0 to $1.3 \mathrm{~kg}$ were used to study the effects of reversible deactivation of motor cortex on movements evoked by microstimulation of PPC. Table 2 provides the details on the number of cooling chips and the placement of each chip in each of the four galagos studied (four cases). In three galagos, two cooling chips were placed in M1 and/or
$3 \mathrm{a}+\mathrm{S} 1$ and, in the other galago, one cooling chip was placed in M1 but covered part of area 3a. All experiments were approved by the Animal Use and Care Committee at Vanderbilt University and conformed to National Institutes of Health guidelines.

Experimental design. Our experimental design and the sequence of steps performed in each experiment are illustrated in Figures 1 and 2. Once the animal was prepared (see below), we rapidly mapped M1 using ICMS to determine the location for the placement of one to two cooling devices (one over a forelimb domain and one over the face domain in M1). We then rapidly explored PPCr using ICMS to find sites that matched those movement representations in M1 over which the cooling devices would be placed. After this, cooling chips were placed over the selected domains in M1. We returned to a site in PPCr that matched the domain of one cooling chip in M1 (e.g., forelimb lift) and then cooled the chip as shown in Figure $2 a$ (the three temperature epochs are described below). After cooling and rewarming of the first chip, we then cooled and rewarmed the second chip while the stimulating electrode remained in place and evoked movements during the different temperature epochs were determined. When this was complete, the stimulating electrode was moved to another site in PPCr and a similar protocol was followed. The details of each portion of the experiment are described below.

Anesthesia and surgical procedures. At the start of each experiment, animals were anesthetized with an intramuscular injection of ketamine hydrochloride (10-30 mg/kg, IM) and intubated. Glycopyrrolate $(0.015 \mathrm{mg} / \mathrm{kg}, \mathrm{IM})$ was given to reduce salivation, ceftiofur sodium $(2.2 \mathrm{mg} / \mathrm{kg}, \mathrm{IM})$ was given as an antibiotic, and dexamethasone $(1 \mathrm{mg} / \mathrm{kg}, \mathrm{IM})$ was given as a prophylactic to prevent swelling of the cortex. Animals were maintained during the first portion of these experiments with the inhalant anesthetic isoflurane (2\%). Throughout the procedures, heart rate, respiration rate, body temperature, and blood pressure were monitored. Later, during microstimulation experiments, after the head post was implanted and the craniotomy was complete, anesthesia was maintained with ketamine hydrochloride $(10-60 \mathrm{mg} / \mathrm{kg} / \mathrm{h})$ diluted with physiological saline (1:4) and delivered intravenously with an infusion pump. This was supplemented every $0.3-2.0 \mathrm{~h}$ with xylazine $(0.2-0.4 \mathrm{mg} / \mathrm{kg}$, IM).

Once anesthetized, animals were placed in a stereotaxic frame, the skin was cut, the temporal muscles were retracted, and the skull was exposed. Several small screws were placed in the skull over occipital cortex and the bases of the screws were secured with dental acrylic. This allowed the head to be secured directly to the stereotaxic frame via a head post secured to the screws with additional dental acrylic. Once the acrylic was set, the eye bars and mouth bar were removed so that the animals face, upper trunk and arms could be observed moving freely during ICMS.

Next, a large craniotomy was made over motor cortex and anterior and posterior parietal cortex. The dura was retracted to expose motor cortical areas M1 and PM, somatosensory cortex, and posterior parietal areas PPCr and PPCc. The exposed cortex was covered with silicone fluid to prevent desiccation. A high-resolution digital image of the exposed cortex was acquired (Nikon D60 camera with a 55-200 mm Nikon lens and a Raynox DCR-250 macro conversion lens) and printed, and all electrode penetrations and the location of the cooling devices (Figs. $1 c, 2 c$ ) were marked on this printed photograph. These regions were then explored using ICMS.

ICMS mapping. Before implantation of cooling devices over M1, cortex was rapidly explored using electrical stimulation delivered through low impedance $(0.5-1.0 \mathrm{M} \Omega$ ) microelectrodes. Trains of pulses were generated by a Master 8 stimulator (AMPI; www.ampi.co.il) with biphasic stimulus isolation (modified BAK BS-I, Bak Electronics; www. bakelectronicsinc.com). Stimuli consisted of long (500 ms) trains of 0.4 ms biphasic pulses (a negative $0.2 \mathrm{~ms}$ phase followed by a positive $0.2 \mathrm{~ms}$ phase) delivered at $300 \mathrm{~Hz}$ and presented every $3 \mathrm{~s}$. Typically, 3-4 stimulation trains were presented at each cortical site. Because the organization of motor cortex has been described previously in different species of galagos using similar techniques (Fogassi et al., 1986; Fogassi et al., 1994; Wu et al., 2000), albeit with short-train stimulation, our goal was limited to determining forelimb domains (e.g., lift, reach, grasp) and face domains in M1. Therefore, for most sites, we used a uniform stimulation 


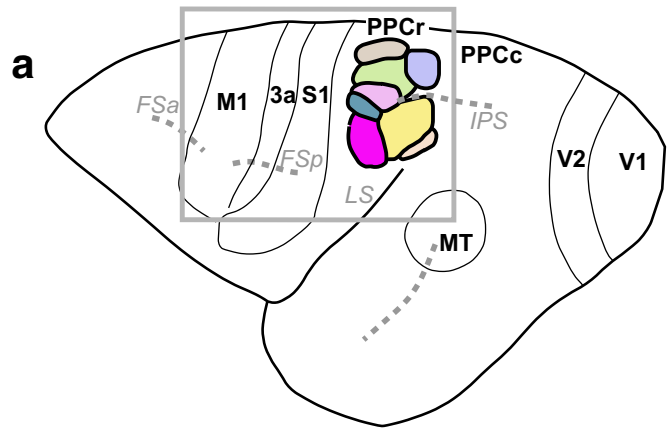

b

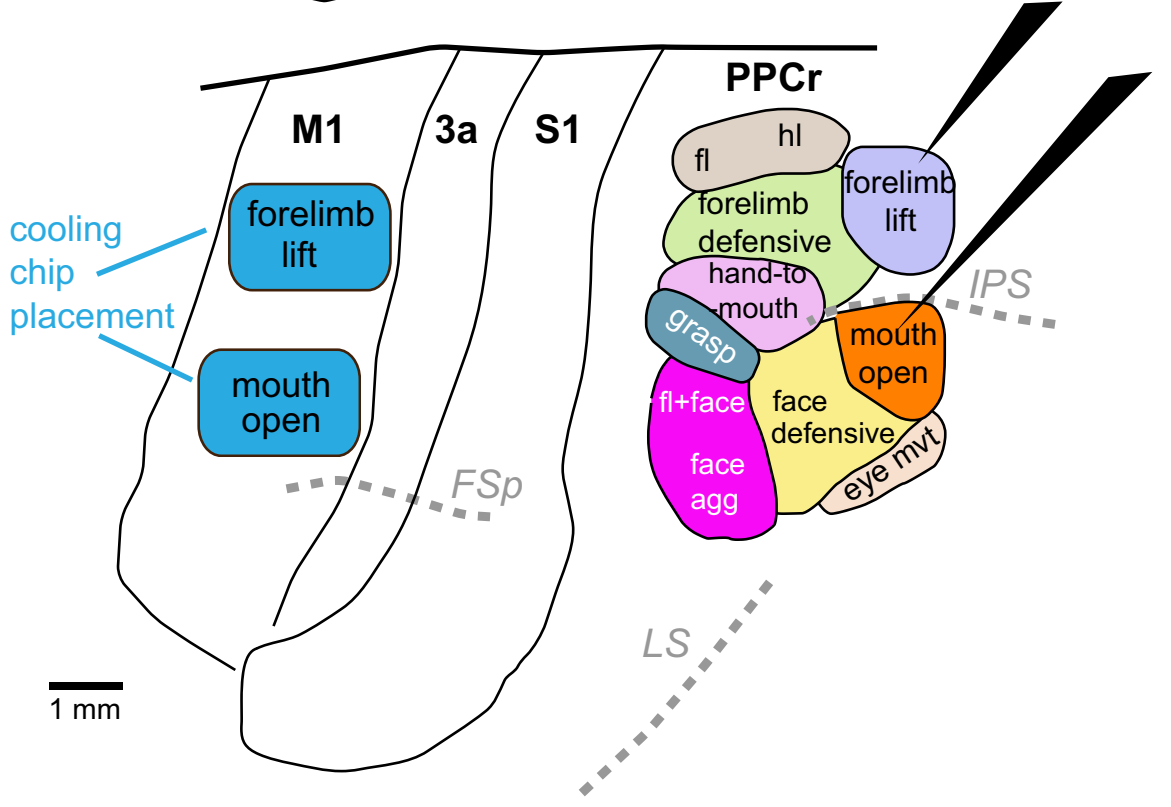

intracortical microstimulation

C

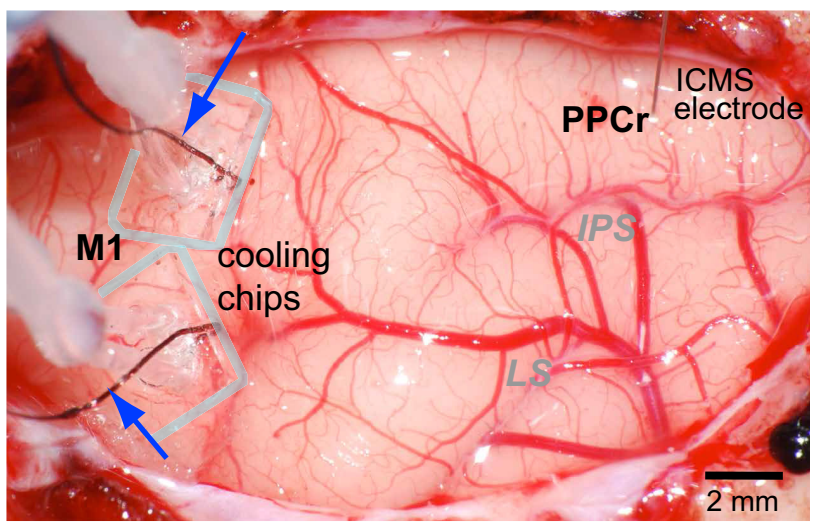

Figure 1. a, Dorsolateral view of the galago neocortex depicting major sulci (italicized abbreviations) and the positions of cortical areas relative to these sulci. The black box, enclosing M1, PPCr, and other fields examined here, indicated the region shown in $\boldsymbol{b} . \boldsymbol{b}$, In this hypothetical example, after exploration of both M1 and PPCr with long-train ICMS, cooling chips (blue rectangles) were implanted over the forelimb-lift and mouth-open domains in motor cortex. In PPCr, representations that corresponded to those over which the cooling chips were implanted were explored using long-train ICMS before, during, and after thermal deactivation in M1. Baseline movements evoked by ICMS in PPCr were compared with those evoked while the forelimb lift or the mouth representation in $\mathrm{M} 1$ were cooled to $10-15^{\circ} \mathrm{C}$ and to movements evoked after $\mathrm{M} 1$ temperature and function were restored. c, Digital image of the exposed neocortex with cooling chips implanted in M1 and a stimulation electrode inserted into PPCr from case 13-36. As the cooling chips are transparent, the outer margin of the PDMS bodies are outlined in gray. The smaller cooling footprint is opaque. Microthermocouples (blue arrows) are embedded in the cooling chips so that cortical temperature can be monitored continuously in both cooling chips throughout the experiment. This image was taken $12 \mathrm{~h}$ after implantation and after multiple cooling and warming epochs. Note that the cortex below the cooling chip has normal vasculature and no damage has been induced by this procedure. Maps of PPCr in $\boldsymbol{a}$ and $\boldsymbol{b}$ are modified from Stepniewska et al., 2005. See Table 1 for abbreviations.

current amplitude of $100 \mu \mathrm{A}$ to explore M1 and did not attempt to determine the current threshold at individual sites. Once we determined the best locations for our cooling chips within M1, we explored the rostral division of PPC (PPCr) with the goal of simply finding movement domains matching those in M1 (as well as domains that did not match; see below). Given its higher ICMS current thresholds, we explored PPCr with higher stimulation current $(200-300 \mu \mathrm{A}$, confirmed by measurement on an oscilloscope via the voltage drop across a $100 \mathrm{k} \Omega$ resistor in series with the stimulation circuit). Because current thresholds for PPCr have been well described for galagos in previous studies (Stepniewska et al., 2005, 2009a), we did not study these in detail. In both M1 and PPCr, the microelectrode was lowered perpendicular to the cortical surface to a 


\section{a experimental design}

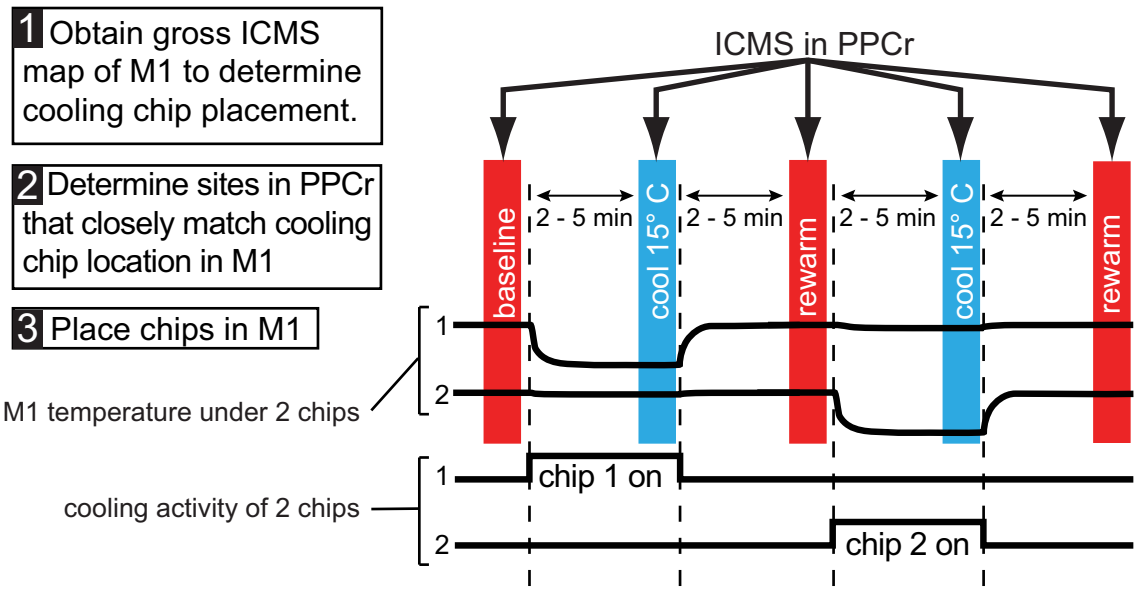

\section{b cooling chip c implanted cooling chip}

\section{footprint}

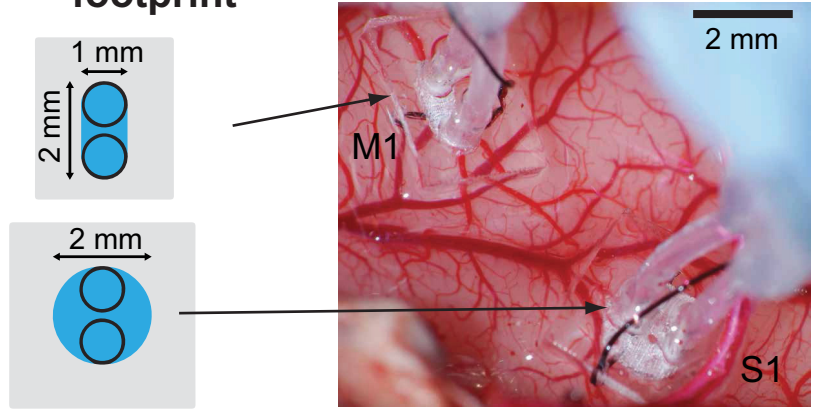

Figure 2. Schematic of our experimental design (a), dimensions of cooling chip footprint (b), and implantation in M1 and S1 in a galago (c). $\boldsymbol{a}$, Experiments started with gross ICMS maps of M1 (Step 1) and PPCr (Step 2). Based on these data, cooling chips were placed at one or two locations in M1 (Step 3). For each ICMS site, we tested stimulation during three epochs (vertical colored bars): baseline, cool (deactivation in M1 or S1), and rewarm (recovery). When two chips had been placed, there were two additional ICMS epochs tested: cooling of the second chip and rewarm. During each epoch, we studied movements evoked by long-train ICMS in PPCr. There was a 2-5 min interepoch interval such that ICMS data collection occurred after the cooled tissue had been stabilized for several minutes at a given temperature. Top black traces, Temperature of cortical surface under each cooling chip. Bottom black traces, Timing of cooling for each chip. Cooling devices have a small footprint $(\boldsymbol{b})$ and are largely transparent when implanted on the cortex (c). The footprints illustrated in care those of the cooling chips implanted in cover the forelimb lift representation in M1 and the tongue representation in $\mathrm{S} 1$ (case 13-37). In c, medial is to the top and rostral is to the right. Conventions are as in previous figure.

depth of 1.5-1.8 mm. Penetration sites were marked on a high-resolution digital image of the exposed cortex. After this exploratory ICMS session, cooling chips were placed over specific representations within M1 (Fig. 1).

Cooling chips. Cooling chips, which contain an integrated microthermocouple to record temperature at the chip/brain interface, were custom fabricated from polydimethylsiloxane (PDMS) and silicone tubing. The design, fabrication, and functional properties of these devices have been described previously (Cooke et al., 2012b). Briefly, cooling chips are operated by pumping chilled ethanol through the tubing; coolant flow is regulated to maintain a hypothermic target temperature in adjacent cortex. Because this initial publication, a new, more compact design fabricated with laser cutting has been developed (Cooke et al., 2014; Goldring et al., 2014; Fig. 2b). Newer chips have smaller cooling footprints but function similarly, albeit more efficiently, requiring lower coolant flow rates to achieve the same cortical temperature and therefore are well suited for deactivating small domains within M1. Because both devices are controlled via thermal feedback, there is no practical difference in operation, although the smaller dimensions of the new design made surgical placement easier, particularly in the relatively small galago brain.
In the newer cooling chips used in this study, ethanol coolant flows through a laser-cut chamber separated from the cortical surface by a $100 \mu \mathrm{m}$ PDMS membrane. This chamber, defining the cooling footprint, ranged from 1.7 to $4.9 \mathrm{~mm}^{2}$ (Fig. 2b).

In these chips, temperature was measured at the cortical surface. We collected thermographic data on our cooling chips to characterize the spread of cooling in vivo using an animal with a similarly sized brain (ferret). Cooling to a chip/cortex interface temperature of $15^{\circ} \mathrm{C}$ produced temperatures of $20^{\circ} \mathrm{C}$ only to a depth of $1500 \mu \mathrm{m}$ below the center of the cooling chip. Because heat transfer drops off dramatically with distance from the cortical surface (Cooke et al., 2012b), fibers of passages, which are located below layer 6 at a depth $>2500 \mu \mathrm{m}$ in M1 of galagos, were unlikely to be exposed to temperatures that would affect their activity (e.g., $29^{\circ} \mathrm{C}$ or lower; see Cooke et al., $2012 \mathrm{~b}$ for electrophysiological recording data and thermal maps). At $1 \mathrm{~mm}$ from the edge of the cooling channel surface, when chip/cortical surface temperature was set at $15^{\circ} \mathrm{C}$, cortical temperatures were $25^{\circ} \mathrm{C}$ at the coldest, with higher temperatures found at depths below this.

Placement of cooling chips. Placement of microfluidic thermal regulators (cooling chips) on specific M1 movement representations was guided by the initial exploration of motor and posterior parietal cortex using techniques described above. Placement relative to architectonic boundaries of cortical fields was later verified histologically (Fig. 3, Table 1). Cooling chips were gently laid over the forelimb and/or mouth/face representations in M1 identified by ICMS. However, post hoc analysis indicated that, in a few instances, the cooling chip also covered a portion of area 3a and S1 Table 2). Dental acrylic secured a flexible steel wire ( $0.9 \mathrm{~mm}$ diameter) to the head post and to the coolant tubes $1-2 \mathrm{~cm}$ above the cooling chips on the cortical surface. Final adjustments to chip position were made by bending the steel wire. The transparent cooling chips were photographed relative to the underlying vasculature on the cortical surface before and after the experiment to confirm that chip location did not change (Figs. 1c, 2c).

Deactivation protocol. Our experimental design is illustrated in Figure 2a. Each cooling chip in M1 (in the three cases in which two cooling chips were implanted) was activated separately and movements evoked from stimulation sites in $\mathrm{PPCr}$ were compared across three temperature epochs corresponding to before, during, and after M1 deactivation. The first was the baseline epoch, during which the M1 cortical temperature was in a normal range $\left(35-36^{\circ} \mathrm{C}\right)$ for exposed cortex without the insulation of the overlying skull. At this time, ICMS was used to evoke a baseline movement at a given site in PPCr. After this, over a period of 1-5 min, coolant was pumped through the cooling chip until a temperature of $15^{\circ} \mathrm{C}$ was reached and stabilized for at least $2 \mathrm{~min}$. In this second epoch ("cooling epoch"), ICMS was again applied to the same PPCr stimulation site and evoked movements were noted. After this, the pump was turned off and the cortex returned to normal temperature $\left(35-36^{\circ} \mathrm{C}\right)$ and was allowed to stabilize for at least $3 \mathrm{~min}$. During this third, rewarm epoch, ICMS was applied for a final time and evoked movements were noted. Throughout all three epochs, the location of the stimulating electrode never changed. In some galagos, two cooling chips were used sequentially in M1 (one in a forelimb domain and one in a face domain). For these cases, ICMS at a given site in PPCr would be applied 


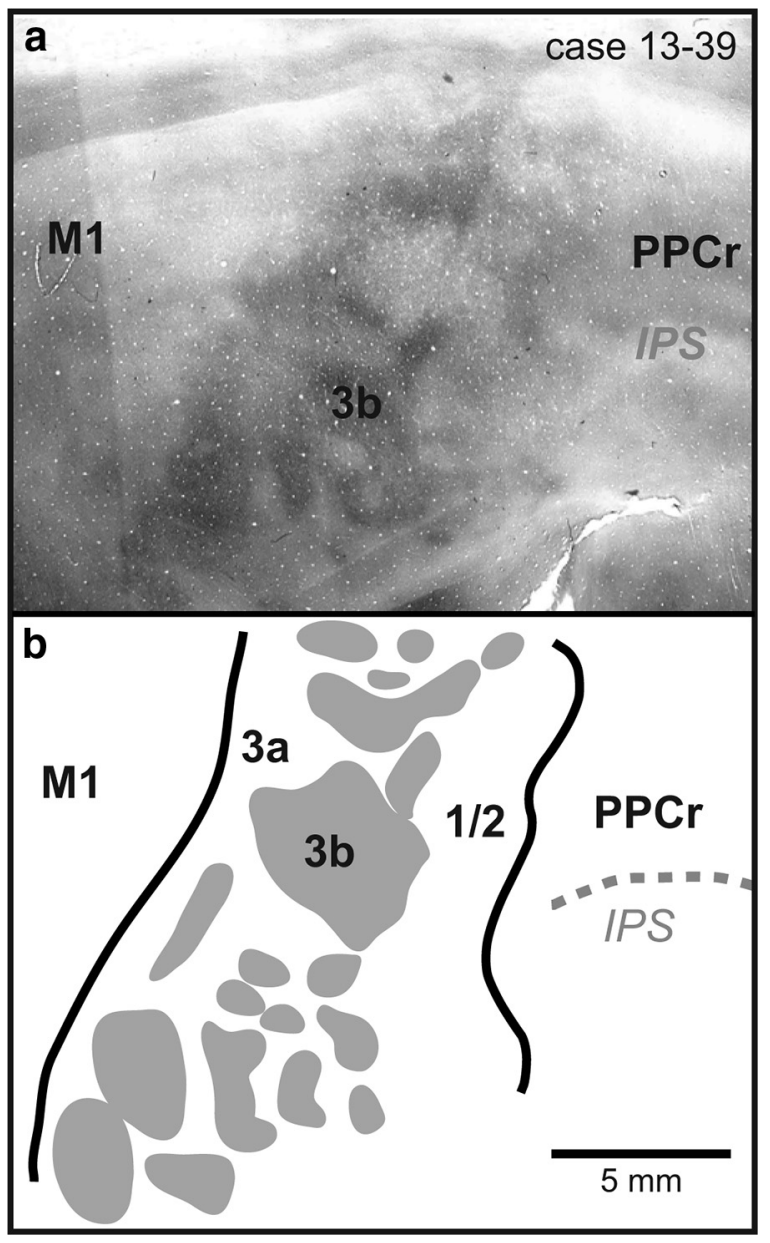

Figure 3. a, Digital image of a single section of cortex that was flattened, sectioned tangential to the cortical surface, and stained for $\mathrm{C} 0$ in case $13-39$. Area $3 \mathrm{~b}$ stains heterogeneously, containing $\mathrm{C} 0$-dark and $\mathrm{C} 0$-light regions. Area $3 \mathrm{a}$ is lightly stained and $\mathrm{M} 1$ and area $1 / 2$ stain moderately for $\mathrm{C} . \boldsymbol{b}$, Reconstruction from the same case generated from the entire series of $\mathrm{CO}$ sections. It should be noted that individual sections reveal only some of the cortical field boundaries and an entire series combined with a series of additional stains such as myelin are used to reconstruct all cortical field boundaries. Thick black lines mark cortical field boundaries. Dotted gray lines are sulci. Myelin/CO dark islands in $\mathrm{S} 1$ are shaded gray. Rostral is to the right and medial is to the top. Conventions are as in previous figures.

during each of the three epochs as described above, first for forelimb domain deactivation and then for face domain deactivation. Movements evoked by ICMS at a given site in PPCr were described in a written record and videotaped for offline analysis (see below). After all deactivation experiments, probes were inserted into the brain at selected sites.

Perfusion and histological processing of tissue. At the end of the experiment, animals were given a lethal dose of sodium pentobarbital $(>80$ $\mathrm{mg} / \mathrm{kg}, \mathrm{IV}$ ) and then were transcardially perfused with $0.9 \%$ saline followed by $2 \%$ paraformaldehyde and then $2 \%$ paraformaldehyde in $10 \%$ sucrose in phosphate buffer, $\mathrm{pH}$ 7.4. Once perfused, the brain was removed from the skull and the cortex was carefully separated from the brainstem and thalamus and manually flattened. The flattened neocortex was placed between two glass slides and left to soak overnight in $30 \%$ sucrose phosphate buffer. The neocortex was sectioned on a freezing microtome at a thickness of $40 \mu \mathrm{m}$. Alternate sections were stained for myelin (Gallyas, 1979) or cytochrome oxidase (CO) (Wong-Riley, 1979; Fig. 3).

Combining histological and ICMS data. Data were analyzed in several ways. First, functional maps of motor and posterior parietal cortex were generated using methods described previously (Stepniewska et al., 2005; Cooke et al., 2012a). Briefly, electrode sites, blood vessels, sulci, cooling chips, and probes were transposed from our high-resolution images onto

\section{Table 1. Abbreviations}

\begin{tabular}{ll}
\hline Cortical fields & \\
3a & Area 3a \\
3b & Area 3b \\
1/2 & Area 1/2 \\
M1 & Primary motor area \\
MT & Middle temporal area \\
PM & Premotor area \\
PPCc & Posterior parietal cortex (caudal area) \\
PPCr & Posterior parietal cortex (rostral area) \\
PV & Parietal ventral area \\
S1 & Primary somatosensory area (Brodmann's area 3b) \\
S2 & Secondary somatosensory cortex \\
V1 & Primary visual area \\
V2 & Second visual area \\
Sulci & \\
FSa & Anterior frontal sulcus \\
FSp & Posterior frontal sulcus \\
IPS & Intraparietal sulcus \\
LS & Lateral sulcus \\
Body parts and movements & \\
agg & Aggressive \\
bilat & Bilateral \\
blk & Blink \\
dwn & Downward \\
f & Face \\
fl & Forelimb \\
flx & Flexion \\
hl & Hindlimb \\
h to m & Hand to mouth \\
mvt & Movement \\
op & Open \\
ret & Retraction \\
sh & Shoulder \\
twd mid & Towards the midline \\
Other & \\
ICMS & Intracortical microstimulation \\
MA & Movement abolished \\
RM & Reduced movement \\
\hline & \\
&
\end{tabular}

a reconstruction drawn in Adobe Illustrator CS6. Architectonic boundaries of cortical fields were determined using the entire series of sections stained for myelin and CO. Outlines of sections, blood vessels, sulci, probes, and cortical field boundaries were drawn for individual sections and then collapsed onto a single reconstruction by aligning blood vessels, probes, and sulci. These data were matched to our ICMS data by aligning blood vessels, sulci, tissue artifacts, and probes. Rough motor maps of cortical fields were made by drawing interpolating lines around sites that evoked similar movements, separating those sites from nearby sites at which stimulation evoked different movements (Cooke et al., 2012b). Therefore, the final reconstruction contained cortical field boundaries, ICMS sites, and the locations and footprints of cooling chips (Figs. 5, 6, 7, 8).

Videocoding and data analysis. Individual video frames captured during ICMS-evoked movements were copied from Quicktime Player 7 Pro and pasted into Adobe Illustrator, where they were traced to corroborate written descriptions recorded during the experiment. Frame-by-frame displacement of landmarks on the forelimb (often a fingertip) was measured so that speed of the evoked movement could be plotted (Figs. 4, 10, 11, 12).

Sites tested during M1 deactivation were categorized based on whether there was an effect of cooling and whether the neural representations at the ICMS site in PPCr matched that of the site of deactivation in M1 (homotopic) or did not match it (heterotopic). See Results for details.

\section{Results}

Seven cooling chips were implanted in four galagos. The placement of the cooling chips over specific complex movement representations (or domains) was determined during ICMS 
Table 2. Effects of cooling and rewarming M1 on PPCr

\begin{tabular}{|c|c|c|c|c|c|c|c|c|}
\hline \multirow[b]{2}{*}{ Placement of cooling chip } & \multicolumn{2}{|l|}{$13-36$} & \multirow{2}{*}{$\begin{array}{l}13-39 \\
\text { Forelimb lift/ } \\
\text { reach M1 (3a) }\end{array}$} & \multicolumn{2}{|l|}{$13-37$} & \multicolumn{2}{|l|}{$13-38$} & \multirow{2}{*}{$\begin{array}{l}\text { Total } \\
7 \text { chips in } 4 \\
\text { animals }\end{array}$} \\
\hline & $\begin{array}{l}\text { Forelimb } \\
\text { lift/reach M1 }\end{array}$ & $\begin{array}{l}\text { Mouth } \\
\text { open M1 }\end{array}$ & & $\begin{array}{l}\text { Forelimb lift + } \\
\text { grasp M1 }\end{array}$ & Tongue $3 \mathrm{a}+\mathrm{S1}$ & $\begin{array}{l}\text { Forelimb } \\
\text { lift/reach M1 }\end{array}$ & $\begin{array}{l}\text { Tongue/grimace } \\
\mathrm{M} 1+3 \mathrm{a}+\mathrm{S1}\end{array}$ & \\
\hline \multicolumn{9}{|l|}{ Cooling M1 } \\
\hline $\begin{array}{l}\text { Number of sites tested in PPCr with } \\
\text { cooling chip in M1 }\end{array}$ & 3 & 3 & 9 & 9 & $\begin{array}{l}0 \text { (not counted: } 9 \text { with } \\
3 \mathrm{a} / \mathrm{S1} \text { cooling) }\end{array}$ & $2^{a}$ & $3^{a}$ & $\begin{array}{c}29 \text { tests }^{b} \text { from } \\
24 \text { sites }\end{array}$ \\
\hline $\begin{array}{l}\text { Homotopic effects/total homotopic } \\
\text { tests (M1 cooling) }\end{array}$ & $2 / 2$ & $0 / 1$ & $5 / 5$ & $6 / 7$ & $\begin{array}{l}\text { (not counted: } 0 / 2 \text { with } \\
3 a / S 1 \text { cooling) }\end{array}$ & $1 / 1$ & $1 / 2$ & $15 / 18(83 \%)$ \\
\hline $\begin{array}{l}\text { Heterotopic effects/total heterotopic } \\
\text { tests (M1 cooling) }\end{array}$ & $0 / 1$ & $1 / 2$ & $2 / 4$ & $0 / 2$ & $\begin{array}{l}\text { (not counted: } 0 / 7 \text { with } \\
3 a / S 1 \text { cooling) }\end{array}$ & $0 / 1$ & $1 / 1$ & 4/11 (36\%) \\
\hline $\begin{array}{l}\text { Distance of heterotopic representation } \\
\text { in M1 from chip edge } \\
\text { Rewarming M1 (among sites where } \\
\text { there was an effect of cooling) }\end{array}$ & $\begin{array}{l}\text { No heterotopic } \\
\text { effects }\end{array}$ & $\begin{array}{l}\text { No exact } \\
\text { match }^{d}\end{array}$ & $\begin{array}{l}0.5 \mathrm{~mm} \\
1.4 \mathrm{~mm}\end{array}$ & $\begin{array}{l}\text { No heterotopic } \\
\text { effects }\end{array}$ & $\begin{array}{l}\text { No heterotopic } \\
\text { effects }\end{array}$ & $\begin{array}{l}\text { No heterotopic } \\
\text { effects }\end{array}$ & $1.5 \mathrm{~mm}$ & \\
\hline Return to baseline & $2 / 2$ & $0 / 1$ & $3 / 7$ & $5 / 6$ & $0 / 0$ & $1 / 1$ & $1 / 2$ & $12 / 19(63 \%)$ \\
\hline Reduced or altered movement & $0 / 2$ & $0 / 1$ & $3 / 7$ & $1 / 6$ & $0 / 0$ & $0 / 1$ & $0 / 2$ & $4 / 19(21 \%)$ \\
\hline No movement & $0 / 2$ & $1 / 1$ & $1 / 7$ & $0 / 6$ & $0 / 0$ & $0 / 1$ & $1 / 2$ & $3 / 19(16 \%)$ \\
\hline
\end{tabular}

${ }^{a}$ When two cooling chips were tested in an animal, all PPCr sites were tested during cooling of both with the exception of one site in this case, which accounts for the mismatch in sites tested for case $13-38$.

bOne ICMS site in PPCr tested for cooling of two cooling chips is counted as two "tests."

'One hypothesis is that heterotopic effects result from spread of cooling effect into additional movement representations in M1 that are homotopic with PPCr-evoked movements affected by cooling. These distances represent the minimum required spread for this to take place.

${ }^{d}$ An exact match for PPCr movement (forelimb retraction) was not observed in M1. A related representation (forelimb lift) in M1 was $800 \mu \mathrm{m}$ from cooling location.

mapping. Cortical field boundaries were later verified using myelin- and CO-stained tissue. The details of the architectonic appearance of cortical fields in parietal and motor cortex of galagos have been described previously (Wu et al., 2000; Wong and Kaas, 2010). Briefly, S1 is particularly striking in sections stained for $\mathrm{CO}$ (Fig. 3) as a heterogeneous field composed of islands of CO dark regions (illustrated in Figs. 3, 4, 5, 6, 7, 8, 9, 10, 11, and 12 with gray shading) on a CO-light background. The pattern of $\mathrm{CO}$ intensity matches the pattern of myelin density, visible in alternate sections stained for fibers. Cortex immediately rostral (area 3a) and caudal (area 1/2) to S1 stains lighter for both myelin and CO. Motor cortex is a relatively darkly myelinated field and PPCr is moderately myelinated.

In all cases, cooling chips were placed over frontal cortex, where ICMS evoked movements but, as confirmed later, not all cooling devices were placed entirely within the borders of M1. Our post hoc identification of cortical field boundaries indicated that four cooling chips in three animals (13-36, 13-37, and 1338) were implanted within the boundaries of M1. In two cases, one cooling chip also covered portions of area $3 \mathrm{a}(13-39)$ or areas $3 a$ and S1 (13-38). Finally, in one case (13-37), one cooling chip was placed in areas $3 \mathrm{a}$ and $\mathrm{S} 1$ and did not cover any portion of M1. In the following results, we first describe the effects of cooling M1 on evoked movements in PPCr. We then describe the effects of rewarming M1 and finally the effects of cooling areas 3a/S1 on evoked movements in PPCr. Across all four animals, 22 sites in PPCr and two sites in area 1/2 were examined during a coolingrewarming cycle at one or two locations in M1 (Table 2). Some the same sites were examined during multiple cooling and rewarming sessions and during sequential cooling of the two cooling chips in a given animal. Therefore, although the number of total sites in our four cases is 22 , the number of "tests" of an effect for cooling one of more chips sequentially is 29 .

\section{Effects of cooling M1 on PPCr}

When examining the effects of reversible deactivation of particular movement representations in M1 on evoked movements in PPCr, the type of effect fell into four distinct categories (Table 2). We term the most common category ( 15 of 18 tests in a homo- topic location to that of the chip placement in M1) a "homotopic" effect (Fig. 4). In this category, deactivation of a movement domain of the forelimb or face in M1 resulted in a complete loss or reduction in amplitude of any movements evoked from matching domains in PPCr. We observed homotopic effects from M1-forelimb cooling (Figs. 4, 5f, 6d, 7d, 9e, 11, 12) and M1-face cooling (Figs. $5 d, 9 c$ ) $83 \%$ of time. Frequently, evoked movements in PPCr were closely matched to the domain deactivated in M1, but were not identical. For example, the forelimb lift/reach representation in M1 was cooled in all four cases; for most of these, this M1 deactivation resulted in a loss or reduction of a highly similar or identical evoked movement in the forelimb lift representations at one or more PPCr sites (Figs. 4, $6 d, 7 d, 9 e, 11$, 12). In the same homotopic effect category, related representations in PPCr were affected by cooling the forelimb lift representation in M1, including forelimb retraction (Fig. 5f), grasp (Fig. $6 d$ ), reach, and hand-to-mouth (Fig. $7 d$ ). All of these movements included the forelimb, but they were not identical to the deactivated domain in M1 (forelimb lift).

The second most prevalent category (7 of 11 tests or 64\%), termed "negative heterotopic effect" (Fig. 4), was often associated with homotopic interactions between M1 and PPCr. In this category, deactivation of a movement representation of the forelimb or face resulted in no change in evoked movements involving different body parts in PPCr. For example, we frequently observed instances in which cooling the M1 forelimb lift domain had no effect on face movements (e.g., grimace and blink, Figs. 5f; $6 d, 9 e$, and/or ear flex, Figs. $6 d, 7 d$ ) evoked from PPCr. Likewise, cooling the M1 domain representing mouth-open had no effect on the forelimb retractions evoked in PPCr (Fig. 5d).

Although our results indicate a strong functional specificity between homotopic domains in M1 and PPCr, there were additional effects of cooling M1 on evoked movements in PPCr that demonstrate that this relationship is more complex. We term a third, less common category (four of the 29 sites examined), a "heterotopic" effect (Fig. 9). In this category, deactivation of a movement representation of the forelimb or face in M1 caused a change in movements evoked in PPCr that involved different body parts (four of 11 tests, or $36 \%$ of the tests 


\section{effect of cooling different M1 locations on PPCr forelimb representation}
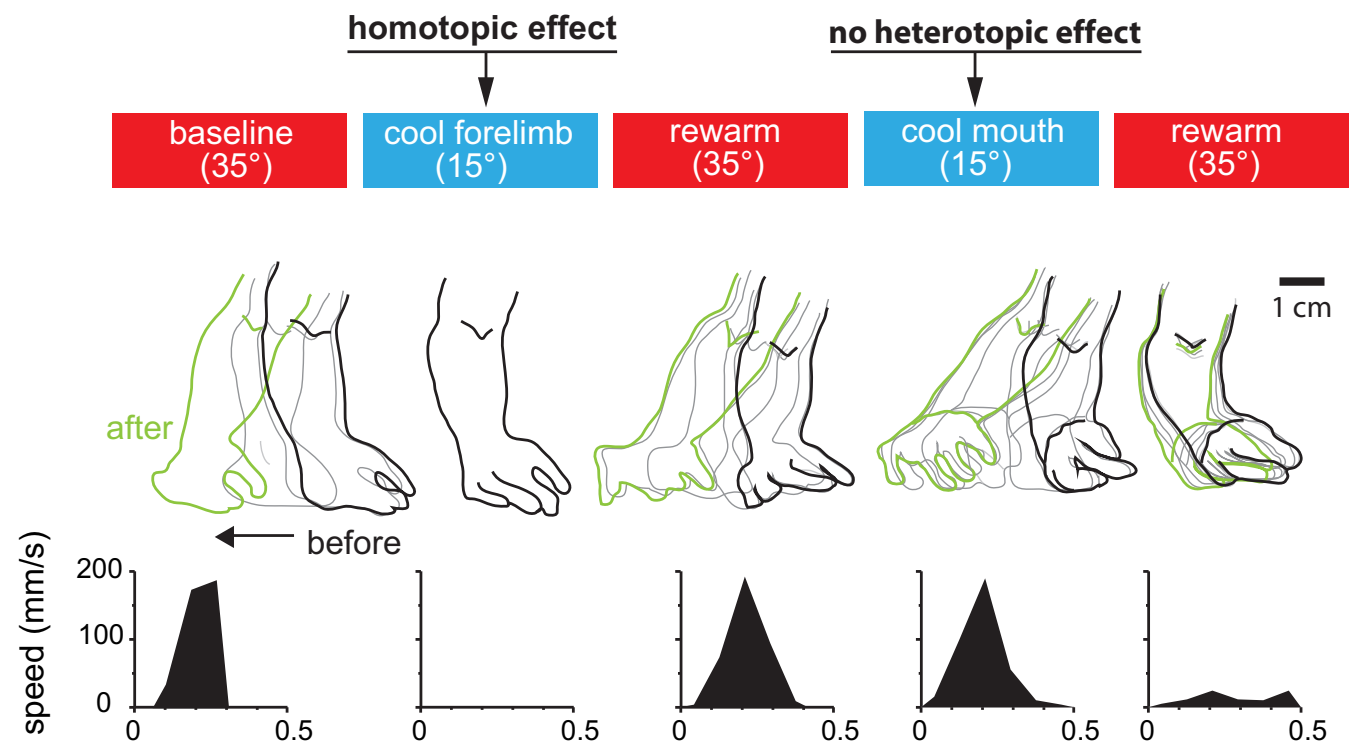

Time after stimulation onset (s)
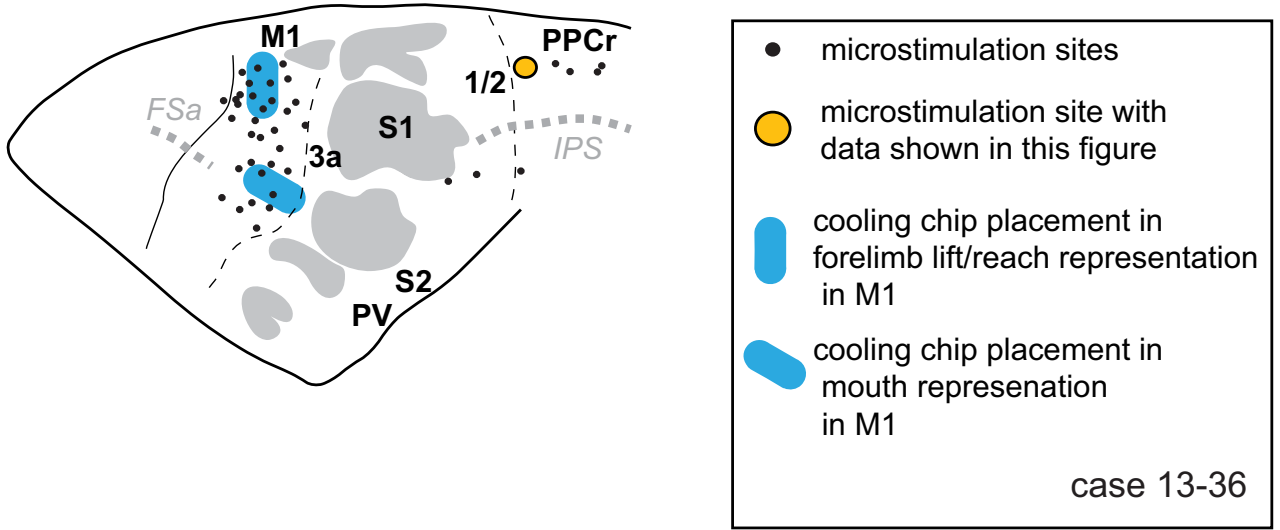

Figure 4. Effect of cooling motor cortex on PPCr stimulation-evoked movements in case 13-36. Arm movements evoked by stimulating a site in PPCr (orange circle) were videotaped before, during, and after cooling deactivation of the forelimb lift/reach or tongue representations in M1. Stimulation epochs are represented by red (baseline warm and rewarm) and blue (cool) rectangles at the top. The outline of the forelimb has been traced for individual video frames during the evoked movement for each epoch. Black tracing is forelimb position before the movement, green is position after the movement, and gray is intermediate positions. Plots illustrate forelimb speed. Cooling the forelimb lift representation in M1 abolished evoked movements in the forelimb lift representation in PPCr, categorized here as a homotopic effect (affecting the same limb). Cooling the M1 tongue representation did not affect evoked movements in the forelimb representation in PPCr; that is, at this site, there was not a heterotopic effect from M1 face to PPCr forelimb.

at heterotopic locations). For example, cooling an M1 mouthopen movement representation abolished ICMS-evoked forelimb lift at a site in PPCr (Fig. 5d). Likewise, cooling a forelimb-lift representation in M1 abolished ICMS-evoked ear flexion and eye blink at sites in PPCr (Fig. 6d). These examples of heterotopic effects in PPCr cannot be explained by the spread of cooling into matching representations in M1. Both our prior testing, noted in the Materials and Methods, and our present data indicate that the spread of cooling effect was minimal. For example, in case 13-39, we observed a heterotopic effect at two sites in PPC (ear flex and blink) when cooling the forelimb lift/reach region in M1/3a (Fig. $6 c, d$ ). Two very close sites in PPC ( $\sim 0.5$ and $1 \mathrm{~mm}$ away), also representing ear flexion and blink, were unaffected by cooling the forelimb lift/reach region in M1/3a. Likewise, in case 13-38 (Fig. 8), a heterotopic effect was seen in the forelimb lift representation in $\mathrm{PPCr}$ when the tongue/grimace representation was cooled (the matching forelimb lift representation in M1 was $\sim 1.5 \mathrm{~mm}$ distant from the edge of the chip; last row of the "Cooling M1" section of Table 2, Fig. 8a). However, when the forelimb lift representation in M1 was cooled, there was not a heterotopic effect of the blink representation in PPCr. The blink representation in M1 was located $\sim 400 \mu \mathrm{m}$ from the edge of the chip. This heterotopic effect at some, but not other, closely situated sites representing the same movement and the lack of heterotopic effect at sites in PPCr the representation of which in M1 is adjacent to the chip edge is not consistent with spread of effect of cooling into adjacent locations in M1.

A final category during cooling was observed at three of the 18 sites $(17 \%)$ in which homotopic effects were tested and was termed a "negative homotopic effect." At these sites, cooling of a specific rep- 

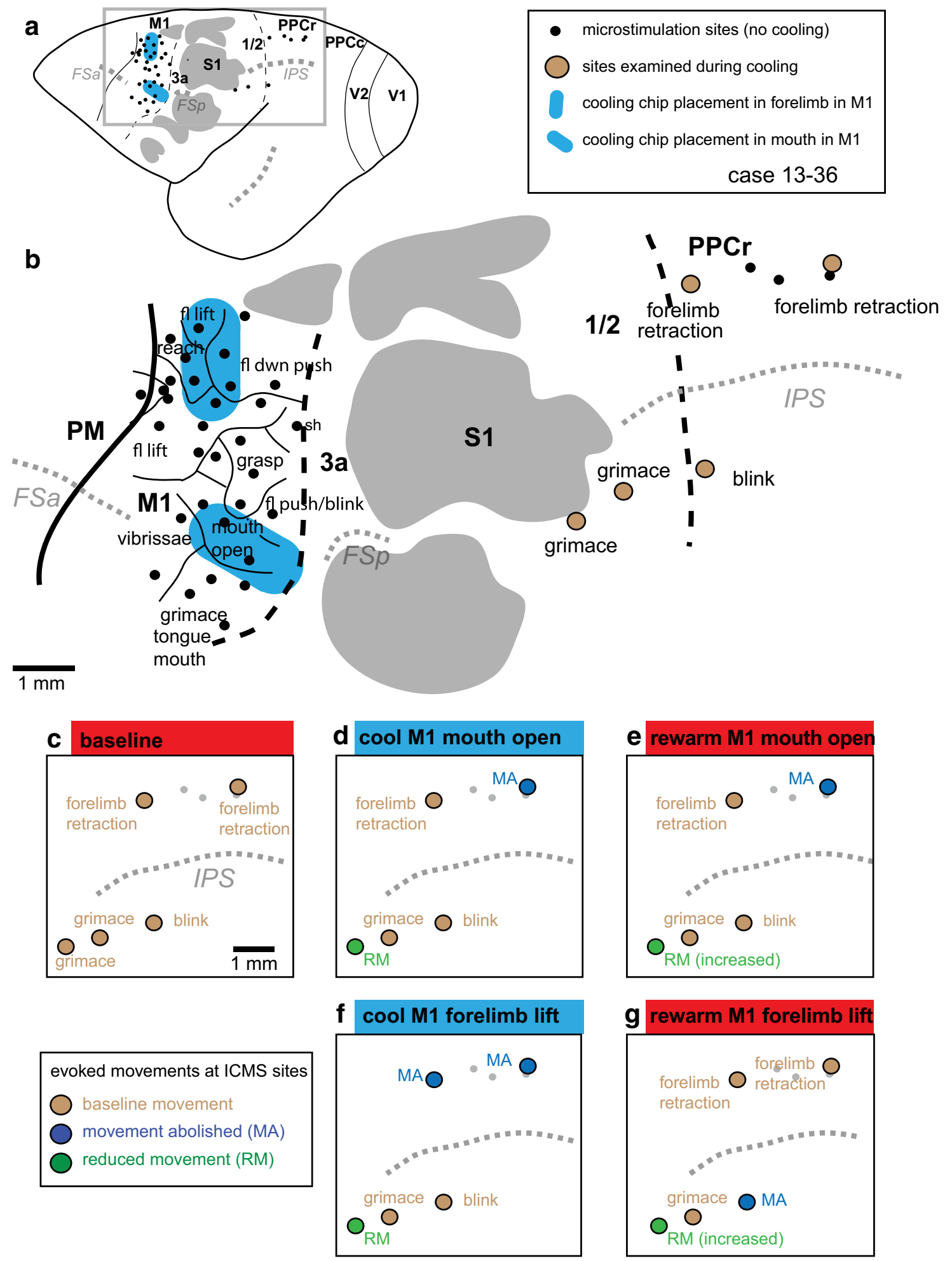

Figure 5. Reconstruction of case 13-36.a, Location of IMCS sites in M1 and PPC r and the placement and size of cooling chips relative to these sites, major sulci, and cortical fields in a whole brain. $\boldsymbol{b}$, Enlargement of boxed area in $\boldsymbol{a}$ with added ICMS maps obtained in M1 and PPCr before cooling regions in M1. In this case, a cooling chip was placed over forelimb representations in M1 (lift, reach, downward push). A second cooling chip was placed over the mouth-open representation in M1. Similar representations were identified in PPCr. $\mathbf{c}-\boldsymbol{g}$, Effects in PPCr during cooling and rewarming of the forelimb and mouth representations in M1. c, Baseline evoked movements in PPCr. $\boldsymbol{d}$, Cooling deactivation of the mouth representation in M1 affects sites in both the mouth (grimace) and the forelimb representation in PPCr.e, Rewarming the mouth representation in M1 resulted in a return of the evoked movement in the mouth representation (although reduced), whereas the evoked movement in the forelimb representation did not return to the baseline condition. Evoked movements were tested at these same sites when the forelimb lift representation in M1 was cooled $(\boldsymbol{f})$ and then rewarmed $(\boldsymbol{g})$. Cooling the forelimb lift representation in M1 abolished evoked movements in the forelimb lift representation in PPCr and reduced evoked movement at one site in the face representation of PPCr. Rewarming this representation in M1 returned movements to normal within the forelimb representation in PPCr. The evoked movement at the one affected site in the face representation increased in amplitude with rewarming, but did not return to baseline levels. Thick black lines mark cortical field boundaries (dashed black lines represent estimated boundaries). Thin black lines mark boundaries of movement representations. Dotted gray lines are sulci. Myelin/C0-dark islands in $\mathrm{S} 1$ are shaded gray. Conventions are as in previous figures. See Table 1 for abbreviations. 
a

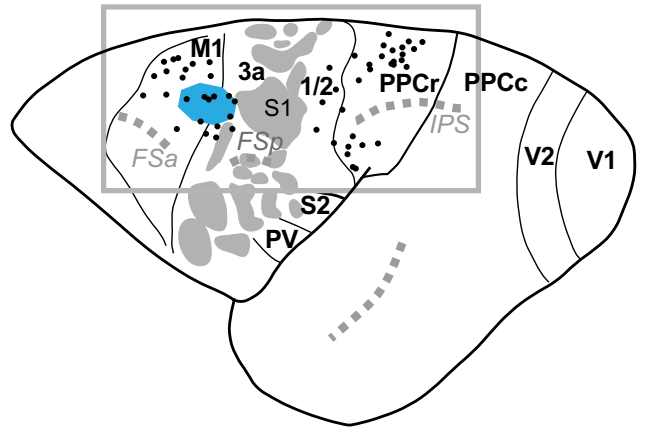
- microstimulation sites (no cooling)
sites examined during cooling
cooling chip placement in $\mathrm{M} 1 / 3 \mathrm{a}$

case $13-39$

b

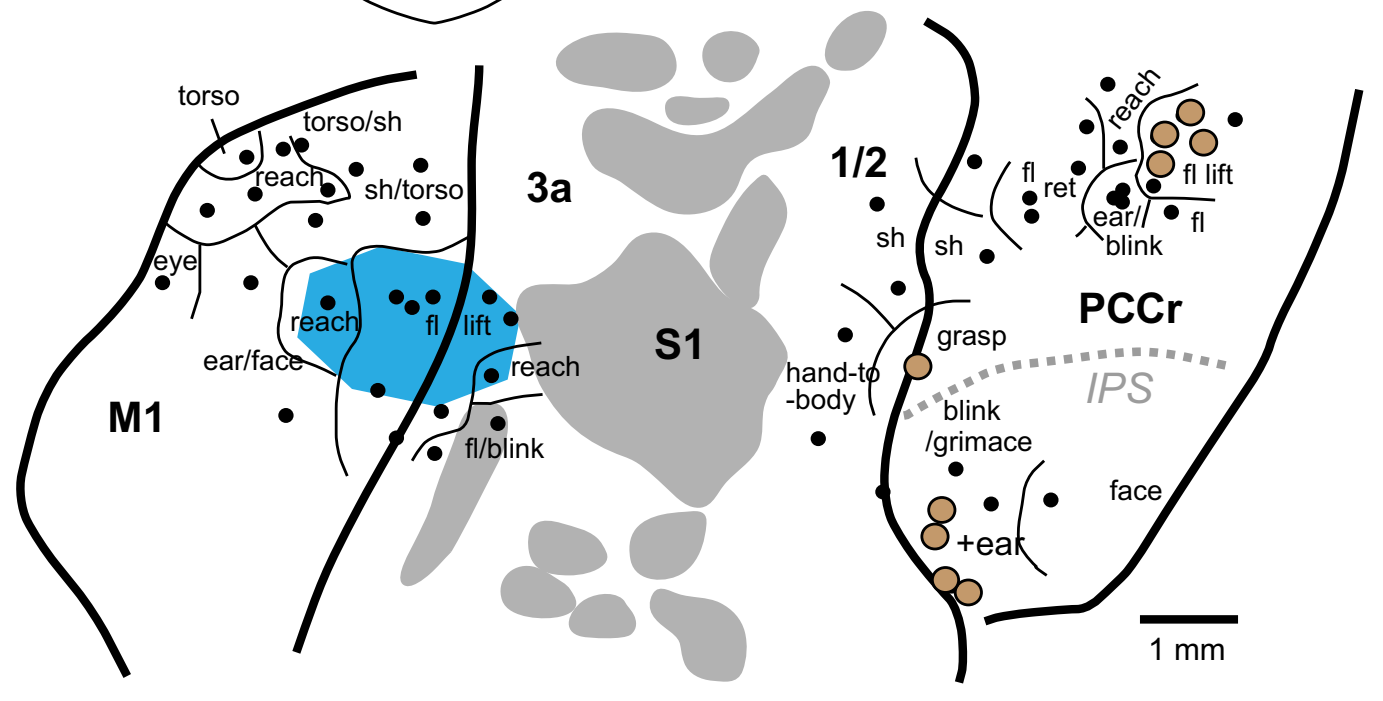

\section{c baseline}

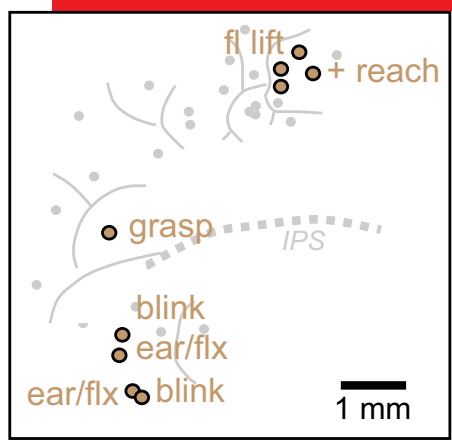

evoked movements at ICMS sites

baseline movement

movement abolished (MA)

reduced movement (RM)
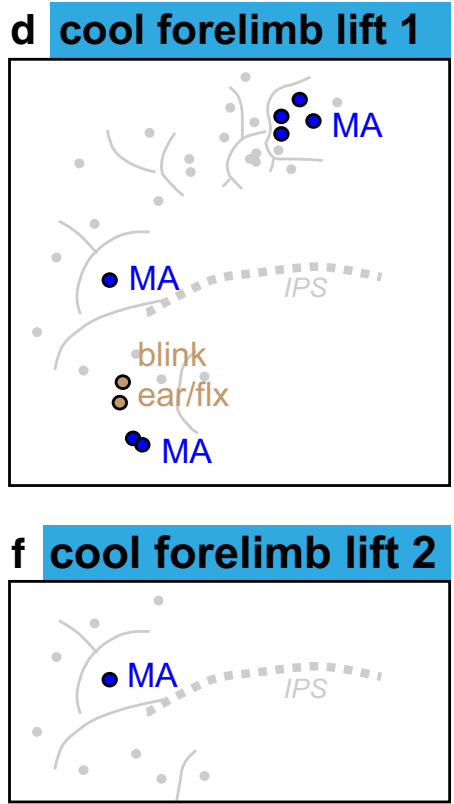
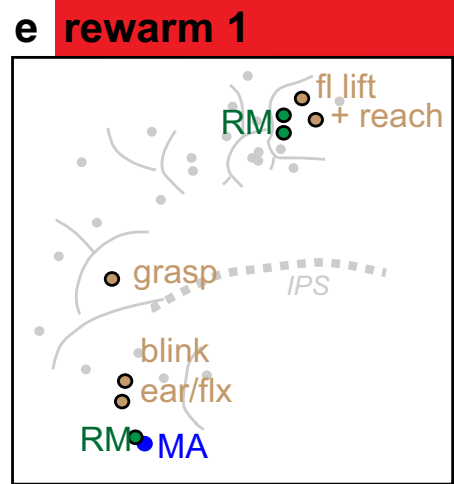

\section{g rewarm 2}

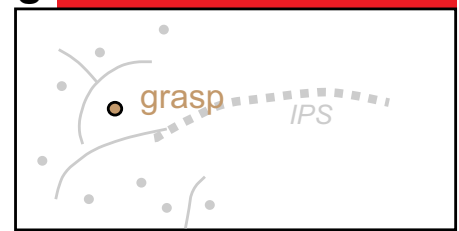

Figure 6. Reconstruction of case 13-39. $\boldsymbol{a}$, Location of IMCS sites, cooling chip, major sulci, and cortical fields. $\boldsymbol{b}$, Enlargement of boxed area in $\boldsymbol{a}$. In this case, the cooling chip was placed over forelimb lift representations in M1, but also covered a small portion of area 3a. c-e, Effects in PPCr during cooling and rewarming of the forelimb lift representation in M1. The effects of cooling the forelimb lift representation in M1 were most profound for similar movement representations (e.g., fl lift, reach) in PPCr, although sites that evoked a grasp movement were also affected (see red and blue sites in the medial portion of $\boldsymbol{c}-\boldsymbol{e}$ ). Interestingly, cooling of the reach, fl lift representations in M1 also affected heterotopic representations in PPCr (e.g., eye blink and ear flexion), but this effect was only for some stimulation sites (see red and blue sites in the lateral portion of $\boldsymbol{c}-\boldsymbol{e})$. At one site (grasp), we cooled and rewarmed twice $(\boldsymbol{f}, \boldsymbol{g})$. We found the same affect (extinction of evoked movement) on both the first and second cooling epochs $(\boldsymbol{d}, \boldsymbol{f})$, as well as a return of movement both times that cortex was returned to its normal temperature $(\boldsymbol{e}, \boldsymbol{g})$. See Table 1 for abbreviations. Conventions are as in previous figures. 


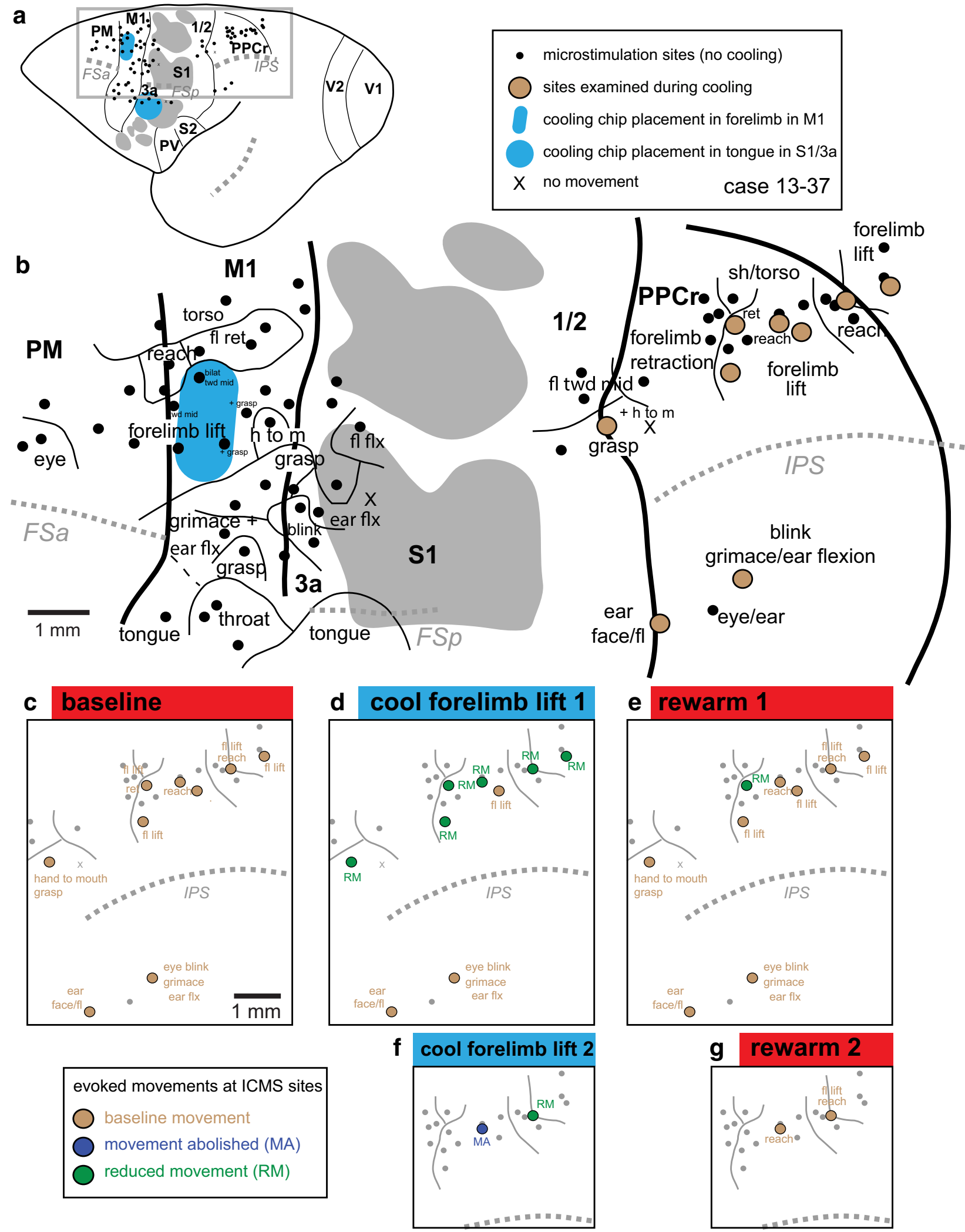

Figure 7. Reconstruction of case 13-37. $\boldsymbol{a}$, Location of IMCS sites, cooling chips, major sulci, and cortical fields. $\boldsymbol{b}$, Enlargement of boxed area in $\boldsymbol{a}$. In this case, the cooling chips were placed over forelimb lift representation in M1 and the tongue representation in S1/3a. Cooling the tongue representation had no effect on any of our tested sites in PPCr. $\mathbf{c}-\boldsymbol{e}$, Effects in PPCr during cooling and rewarming of the forelimb lift representation in M1. Here, cooling in M1 affected all related PPCr sites (forelimb lift, reach, hand to mouth; $\boldsymbol{d}$ ) except one and affected no unrelated sites (e.g., eye blink, ear flexion; $\boldsymbol{d})$. In this case, at two sites, reduction or abolition of evoked movements were replicated in a second cooling and rewarming cycle $(\boldsymbol{f}, \boldsymbol{g})$. Many evoked movements in both M1 and PPCr were more complex than described in $\boldsymbol{b}-\boldsymbol{g}$ and included smaller movements of other body parts. However, for clarity in illustrating, these are only noted for sites under the cooling chip and for sites tested in PPCr. Conventions are as in previous figures. 


\section{a cooling chip placement}
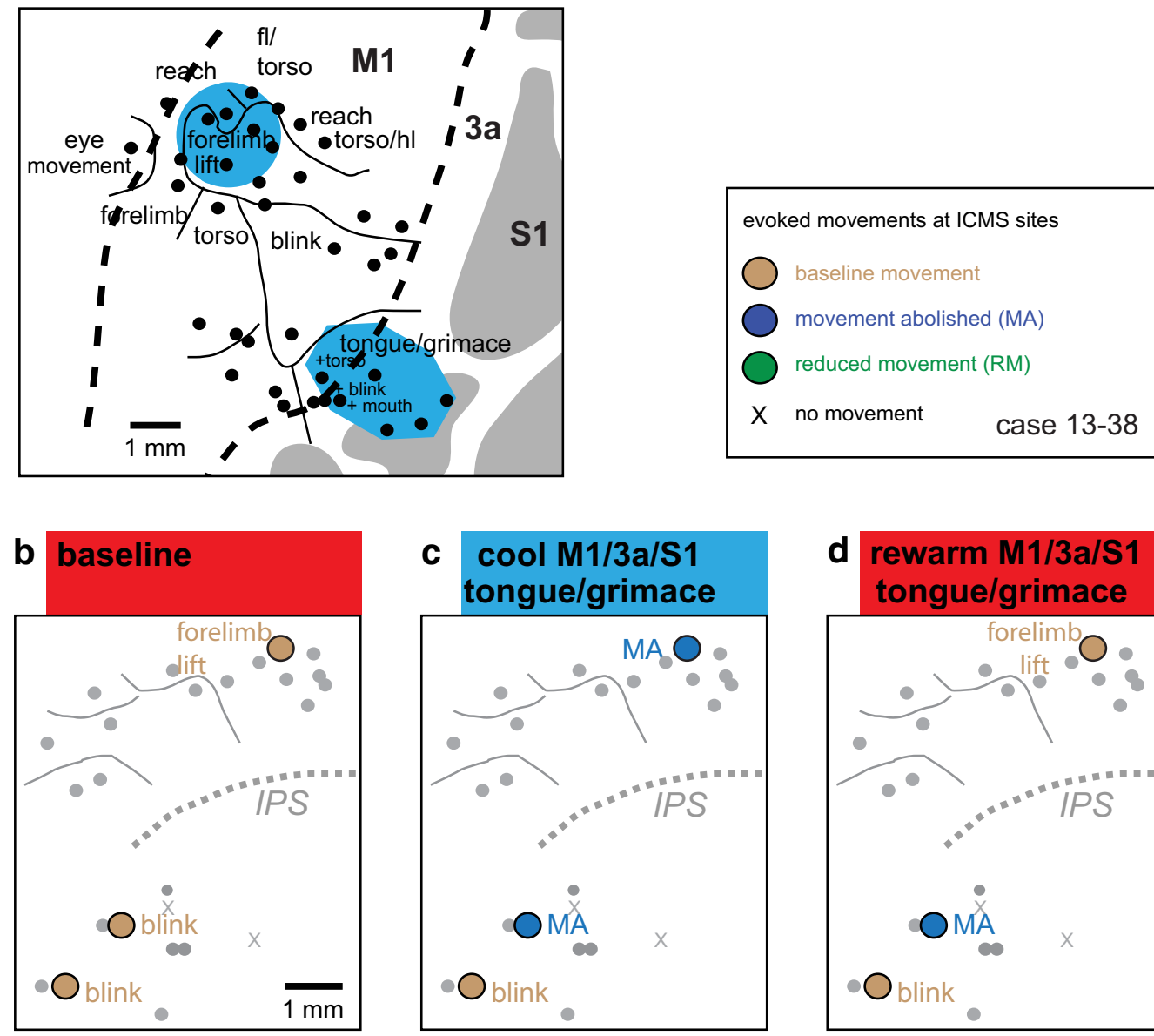
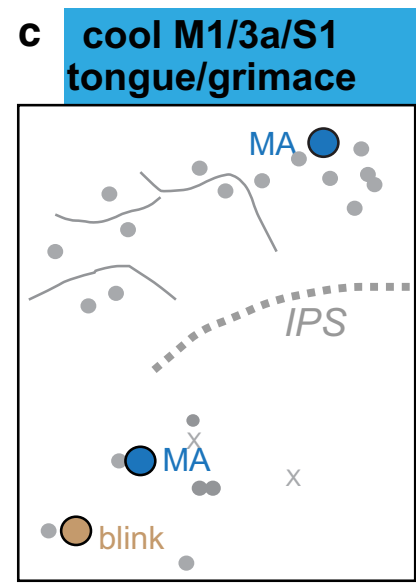

e

\section{cool M1 forelimb lift}

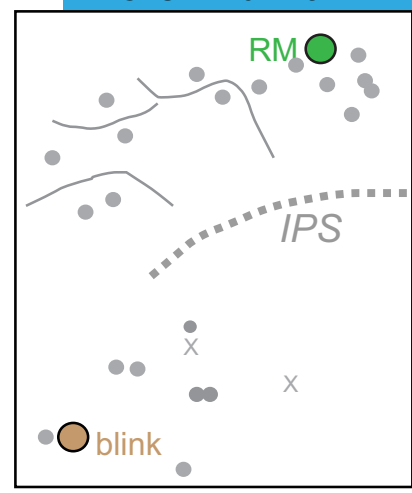

d rewarm M1/3a/S1 tongue/grimace

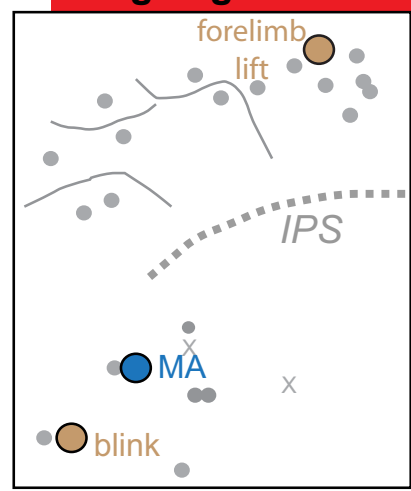

f

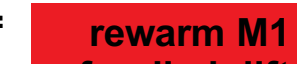
forelimb lift

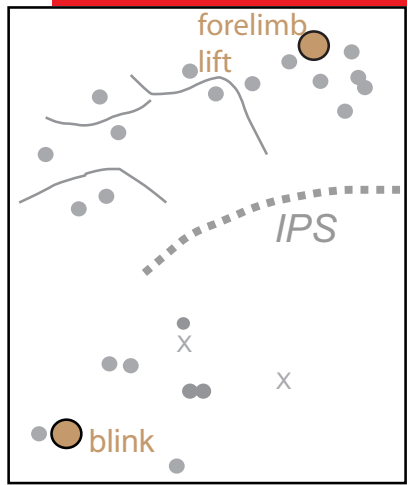

Figure 8. Reconstructions of data from case 13-38. $\boldsymbol{a}$, Enlargement of the area in which cooling chips were placed in the M1-forelimb-lift representation and the tongue/grimace representation in $\mathrm{M} 1 / 3 \mathrm{a} / \mathrm{S} 1$. The results of cooling and rewarming these representation on evoked movement in PPCr are illustrated in $\boldsymbol{b}-\boldsymbol{f}$. Conventions are as in previous figures.

resentation in M1, such as forelimb lift, had no effect on a homotopic movement evoked in PPCr (Fig. 7d).

The proportion of positive homotopic tests $(15 / 18,83 \%)$ was more than twice that of positive heterotopic tests $(4 / 11$, $36 \%)$, a significant difference ( $p=0.017$, Fisher's exact test). Therefore, there appear to be strong interactions between the same or similar pairs of movement representations in M1 and PPCr such that abolishing activity in M1 disrupts movements in PPCr in homotopic domains. Interestingly, there are also more limited effects (36\%) on unrelated domains.

\section{Effects of rewarming M1}

Here, we include only those 19 sites in which PPCr movements were affected by M1 cooling (homotopic and heterotopic effects; Table 2). For most of the sites in which alterations in evoked movements in PPCr were noted, rewarming M1 resulted in a return to the baseline movement (12 of $19 ; 63 \%)$ or a movement with a larger or smaller amplitude (four of $19 ; 21 \%$ ), for a total of 16 of 19 sites returning to normal or near normal baseline conditions (84\%). Figure 10 shows an example of such a movement with an amplitude greater than baseline during the rewarm epoch. In this case, the baseline move- 
heterotopic effect of cooling M1

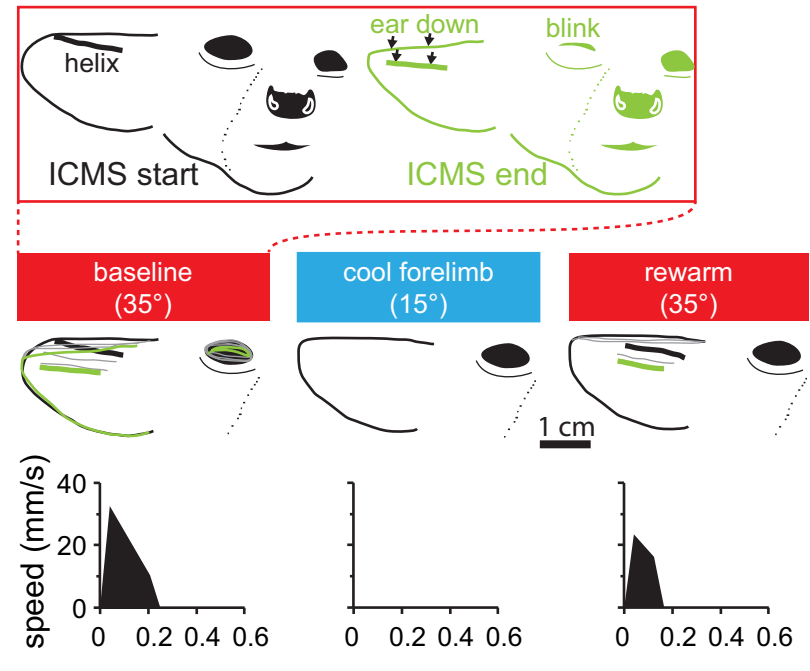

Time after stimulation onset

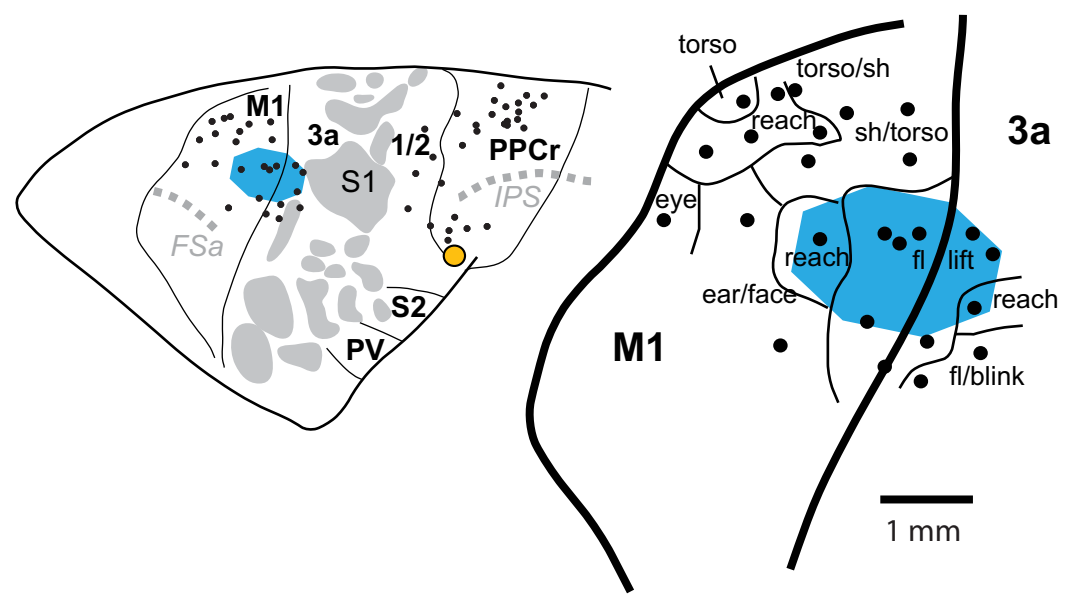

- microstimulation sites

microstimulation site with data shown in this figure

cooling chip placement in forelimb lift/reach represenation in $\mathrm{M} 1 / 3 \mathrm{a}$

Case 13-39

ined during the cooling of the tongue representation in S1/3a. At all sites in PPCr, there was no change in the evoked movements as a result of S1/3a cooling (Fig. 11, middle row). This includes PPCr-evoked movements of both the forelimb and face. This negative effect allowed us to better interpret our results from the two cases in which our cooling device was placed in M1, but also included portions of area 3a (Figs. $6 b, 8$ ). Because there was no effect on evoked movement in PPCr when area $3 \mathrm{a}+\mathrm{S} 1$ was cooled, the effects that we did see in PPCr during cooling $\mathrm{M} 1+3 \mathrm{a}$ can be attributed to the deactivation of M1.

\section{Consistency of effects}

To test the consistency of our effects and to rule out anesthetic instability as the source of observed changes in ICMSevoked movements, we examined some of the same sites in PPCr on a second cooling and rewarming epoch (Figs. 6, 7). In the first case (13-39), a grasping movement evoked in PPCr (Fig. 6c, left of center) was abolished during cooling of the forelimb lift/reach representation in M1 (Fig. 6d). Rewarming M1 resulted in a return to the baseline movement (Fig. 6e). A second cooling of the forelimb lift representation in M1 resulted in the movement being abolished again (Fig. 6f); after rewarming, the baseline movement returned again (Fig. $6 g$ ). A similar finding was observed in case 13-37 (Figs. 7, 12), when a forelimb retraction movement in PPCr was abolished during cooling the forelimb lift representation in $\mathrm{M} 1$ and returned to baseline upon rewarming (Fig. 11, top row). A subsequent cooling-rewarming cycle replicated this result (Fig. 11, bottom row).

\section{Discussion}

Using cooling deactivation, we consistently and reversibly deactivated specific regions of motor cortex and examined the effect on evoked movements in PPCr during multiple cooling-rewarming cycles. Only one other study deactivated M1 and examined the effects on evoked movements in PPC, in this case, in galagos, squirrel monkeys, and owl monkeys

ment was a grasp that was abolished during cooling of the M1forelimb-lift representation. Rewarming resulted in a return of the grasp movement, but with a larger amplitude. At only three sites (of 19 with a cooling effect) in the four animals studied was there no recovery of movement after rewarming of the cortex.

\section{Cooling S1/area 3a}

In one case, the cooling chip was placed over a location that evoked tongue movements in areas $3 \mathrm{a}$ and $\mathrm{S} 1$ (Fig. 7). In this case, the sites in PPCr examined during forelimb-lift cooling in M1 were also exam-
(Stepniewska et al., 2014). Unlike the current investigation, they used muscimol, a $\mathrm{GABA}_{\mathrm{A}}$ agonist, the effects of which can persist and vary continuously for many hours as a result of diffusion to neighboring tissue as well as decreasing deactivation efficacy as the concentration declines volumetrically and through uptake (Hikosaka and Wurtz, 1985, see their Fig. 3 for an example of change across 7 h; Martin, 1991; Martin and Ghez, 1993; Malpeli, 1999).

The most common effect of muscimol deactivation of M1 was a reduction of ICMS-evoked movements in PPCr and higher 


\section{homotopic effect of cooling M1}

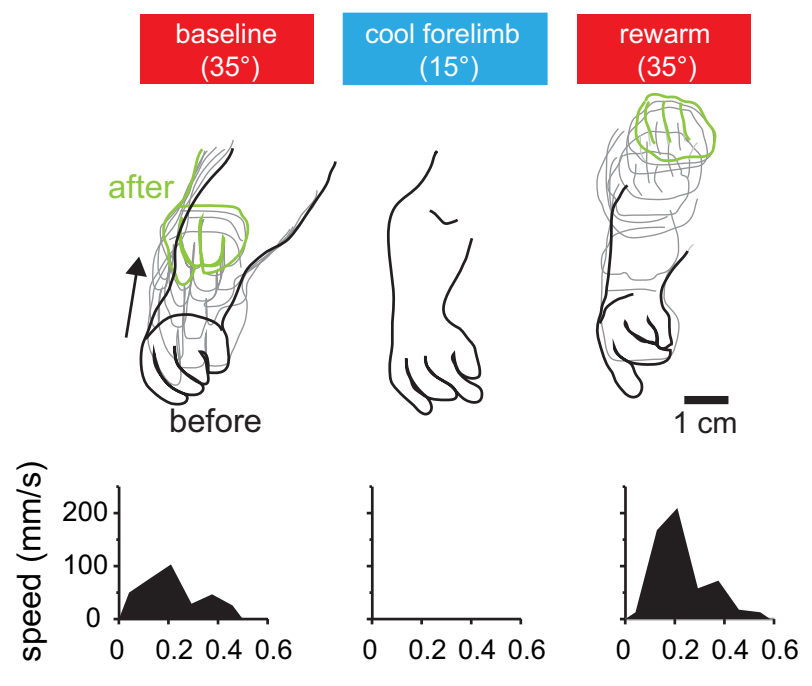

Time after stimulation onset

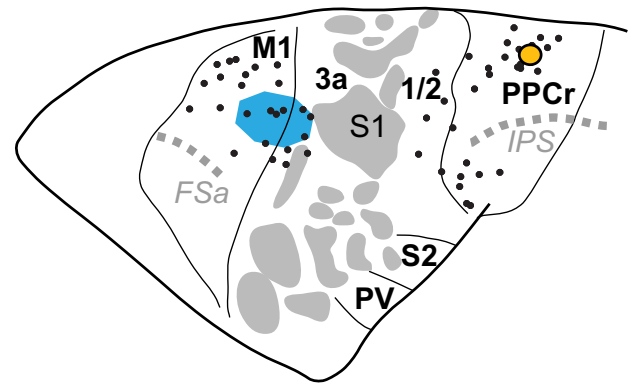

- microstimulation sites

microstimulation site with data shown in this figure

cooling chip placement in forelimb lift/reach represenation in $\mathrm{M} 1 / 3 \mathrm{a}$

Case 13-39

Figure 10. Effect of cooling motor cortex on PPCr stimulation-evoked movements in case 13-39. Arm movements evoked by stimulating a site in $\mathrm{PPCr}$ (orange circle) were videotaped before, during, and after cooling deactivation of the forelimb lift representation in M1/3a. This figure demonstrates homotopic interactions between $\mathrm{M} 1$ and $\mathrm{PPC}$ in which a similar movement evoked in PPCr is abolished. When cortex was returned to normal temperature, the movement returned, the amplitude of the movement was greater, and the end position of the movement was higher than baseline. Conventions are as in previous figures.

stimulation-current thresholds (Stepniewska et al., 2014). In galagos, this effect was restricted to homotopic locations with little effect on evoked movements in heterotopic locations (even with a relatively large muscimol injection, see Fig. $3 a$ of Stepniewska et al., 2014), whereas in owl and squirrel monkeys, additional heterotopic effects in PPCr were observed. In the present study, the most common effect was a loss (rather than a reduction) of evoked movements in homotopic representations in PPCr and no effect on movements in heterotopic representations. Therefore, our results were consistent with previous observations in galagos. The subtle differences observed could be due to differences in the geometry of deactivation from muscimol versus cooling. For example, diffusion of muscimol laterally and into superficial and deep layers of the neocortex may be different than the lateral and laminar effects of cooling. Despite these dif- ferences, both studies demonstrate that deactivation of M1 in galagos reduced or abolished movements in homotopic and related domains in PPCr and had a limited effect on evoked movement in heterotopic locations. Therefore, there appears to be a strong functional relationship between matched and closely matched domains in motor cortex and PPCr, an observation that is supported by a recent optical imaging study in galagos in which ICMS in PPCr activated strictly homotopic locations in M1 (Stepniewska et al., 2011).

To understand these observed effects in PPCr, we must consider the cortical circuits involved in generating complex movements in galagos and other primates. PPCr (and divisions of PPC in other primates) receives direct, dense input from $\mathrm{M} 1$ in galagos and New World and Old World monkeys (Jones et al., 1978; Burman et al., 2008; Stepniewska et al., 2009b; Gharbawie et al., 2011a; Gharbawie et al., 2011b; Bakola et al., 2013). PPCr also projects to M1 in galagos and other primates (Jones et al., 1978; Strick and Kim, 1978; Ghosh et al., 1987; Stepniewska et al., 1993; Gharbawie et al., 2011a; Gharbawie et al., 2011b). PPCr receives direct and indirect input from somatosensory areas and visual extrastriate areas in a number of primates (for review, see Kaas and Stepniewska, 2015). The patterns of connectivity, deactivation studies and studies of intrinsic functional interactions within motor networks led Kaas and Stepniewska (2015) to propose that intrinsic PPCr connections serve to inhibit heterotypic interactions and that the circuitry of matching domains in $\mathrm{M} 1$ and $\mathrm{PPCr}$ contributes to the choice of one behavior over the other. This decision process starts in $\mathrm{PPCr}$, which receives direct and indirect input from somatosensory and visual areas; these inputs drive one domain over the others and suppress competing behaviors represented in adjacent domains. The most active domain in $\mathrm{PPCr}$ then initiates a similar process of selectively activating matching domains in premotor cortex and M1.

The differences in homotopic and heterotopic effects of deactivation in galagos compared with New World monkeys and tree shrews (Baldwin et al., 2014) indicate that this frontoparietal network has been altered with the expansion of PPC in primates (Fig. 12). In tree shrews, a group closely related to primates, we observed only homotopic effects on PPC when M1 was deactivated, suggesting the presence of strong homotopic connections between a relatively simply organized map of movement domains in PPCr and M1 (Remple et al., 2007). In prosimian galagos, the limited number of sites in which we observed heterotopic effects or no effect on a homotopic representation suggests an intermediate pattern of divergent and convergent connectivity between M1 and PPCr, and the greater heterotopic effects in squirrel and owl monkeys suggests a more complex pattern in simians. We would predict that, in Old World monkeys, a more intricate plan of convergence and divergence exists and coevolved with the addition of movement domains to this network. Although connections of PPC and motor and premotor cortex have been examined in macaque monkeys (Lewis and Van Essen, 2000; Gharbawie et al., 2011b; Bakola et al., 2013), specific connections between ICMS-identified domains in PPC have not been studied. Such evolutionary changes to a basic network could account for the increased degrees of freedom for reaching and grasping in simians compared with prosimians and tree shrews.

This idea is consistent with theories of motor control in invertebrates, nonmammalian vertebrates, and mammals, which hold that motor actions and movements are composed of fundamental building blocks or "primitives" and the entire movement repertoire consists of applying a set of operations 


\section{homotopic interactions between $\mathrm{M} 1$ and $\mathrm{PPCr}$}

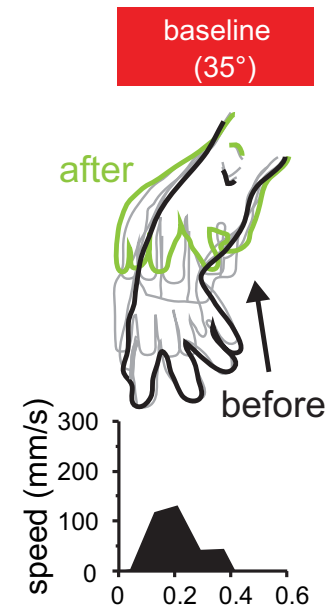

\section{cool forelimb $\left(15^{\circ}\right)$}
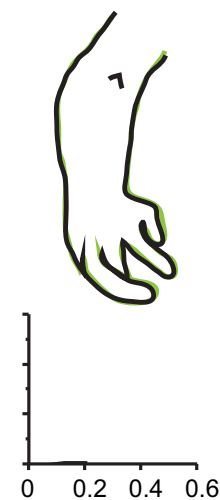

Time after stimulation onset (s)

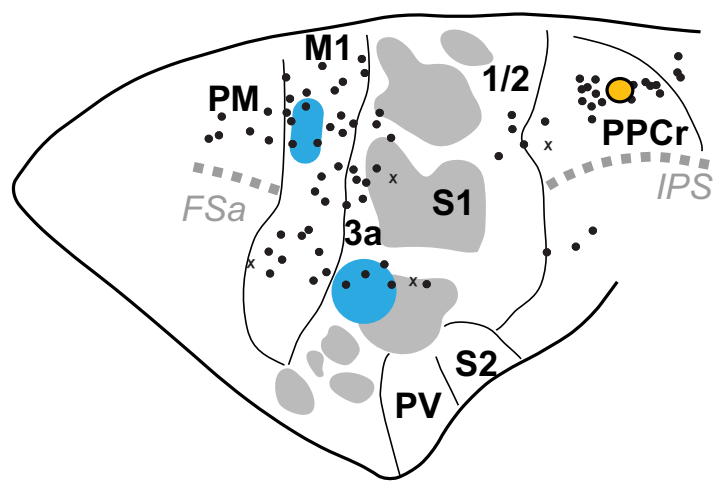

- microstimulation sites

microstimulation site with data shown in this figure

cooling chip placement in forelimb lift representation in $\mathrm{M} 1$

cooling chip placement in tongue represenation in $\mathrm{S} 1 / 3 \mathrm{a}$

x no movement

case 13-37

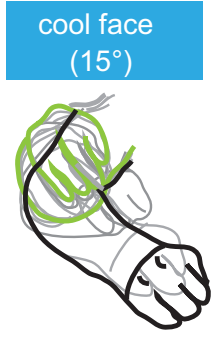

है

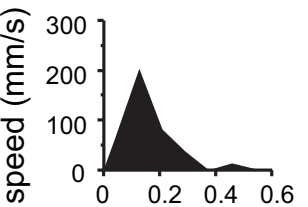

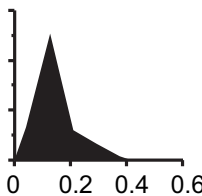

Time after stimulation onset (s)

\section{cool forelimb}

$\left(15^{\circ}\right)$
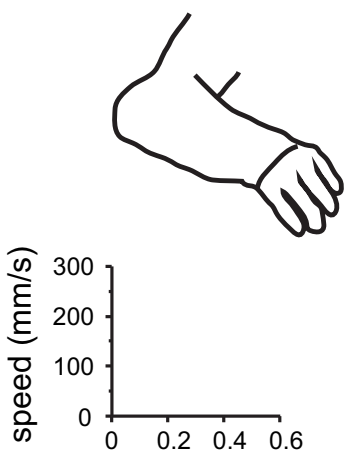

Time after stimulation onset (s)

Figure 11. Effect of cooling motor cortex on PPCr stimulation-evoked movements in case 13-37. Arm movements evoked by stimulating a site in PPCr (orange circle) were videotaped before, during, and after cooling deactivation of the forelimb lift representation in M1 and the tongue representation in $3 \mathrm{a} / \mathrm{S} 1$. The top row shows results when cooling and rewarming the forelimb lift representation in M1, the middle row shows the results when cooling and rewarming the tongue representation in area 3a/S1 ("cool face"), and the bottom row shows the results $\mathrm{M} 1$ was cooled and rewarmed a second time. For both M1-forelimb cooling cycles (first and third rows), stimulation movement was nearly or completely abolished during cooling of the M1 forelimb lift representation and recovered after rewarming of $\mathrm{M} 1$. Cooling the tongue representation in areas $3 \mathrm{a} / \mathrm{S} 1 \mathrm{had}$ no effect on evoked movements at this site. The brain at the left shows the position of cooling chips and the ICMS in PPC in this case. Conventions are as in previous figures. 
a simple homotopic connections (tree shrew) motor output

no

movement

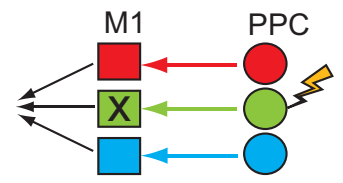

b intermediate divergence/convergence (galago)

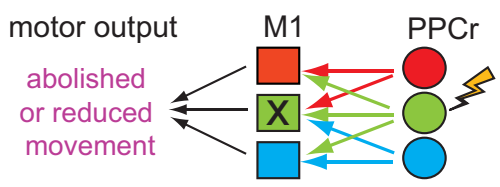

C complex divergence convergence + increase in domains and cortical fields (macaque)

multiple

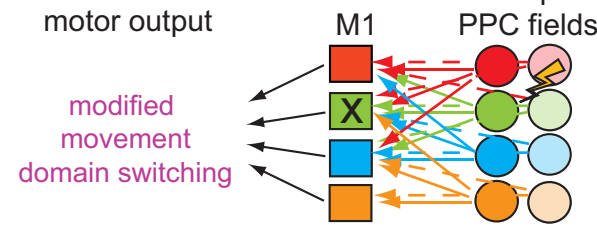

\begin{tabular}{|lcc|}
\hline domains & projections & X inactivate \\
$\square \bigcirc$ reach & with cooling \\
$\square \bigcirc$ grasp & SICMS \\
$\square \bigcirc$ hand-to-mouth & $=$ & \\
$\square \bigcirc$ defensive $\longleftarrow=$ &
\end{tabular}

Figure 12. Schematic illustrating simple $(\boldsymbol{a})$, intermediate $(\boldsymbol{b})$, and complex $(\boldsymbol{c})$ motorposterior parietal networks. Nondivergent, homotopic projections from PPC to M1 are hypothesized for tree shrews (as in $\boldsymbol{a}$ ) and divergent projections from movement domains in different areas in $\mathrm{PPC}$ to $\mathrm{M} 1$ are hypothesized for the macaque monkey $(\boldsymbol{c})$. For example, when a given domain in $\mathrm{M} 1$ is deactivated via cooling (e.g., $X$ in grasp domain in $\mathrm{M} 1$ ), in a simple nondivergent network ( $\boldsymbol{a}$ ) stimulation of a similar domain in PPC would result in no movements. A very complex network consisting of divergent and convergent connectivity from multiple domains and multiple cortical fields (c) would have a different motor output, perhaps including domain switching or modified movements. We hypothesize that galagos have networks of intermediate complexity $(\boldsymbol{b})$.

and transformations to these primitives by combining them in many different ways according to a well defined set of rules (Flash and Hochner, 2005). In mammals, it has been proposed that specific domains in motor cortex access subcortical modules that organize these primitives to generate complex behaviors (Kaas and Stepniewska, 2015). Here, we suggest that basic circuits exist between $\mathrm{M} 1$ and PPC representing specific motor behaviors and, like modules in the spinal cord, these can be considered as "primitives" in the neocortex. These cortical primitives likely exist in all eutherian mammals, including those with relatively small brains such as rats and tree shrews (Fig. 12).

It should be noted that both M1 and PPCr project directly to cervical and thoracic levels of the spinal cord in primates, but the density of these projections varies from relatively dense in Old World monkeys (Murray and Coulter, 1981; Nudo and Masterton, 1990; Galea and Darian-Smith, 1994), to moderate in New World monkeys (Nudo and Masterton, 1990), to relatively sparse in the galagos (Goode and Haines, 1975; Wu et al., 2000). Therefore, electrical stimulation in PPCr after deactivation of M1 could activate this descending pathway directly, which could allow for ICMS-evoked movements in PPCr without a functioning M1. In the present study, however, only a few sites showed a negative effect in homotypical locations of PPCr after deactivation of M1, suggesting that the corticospinal connections of PPCr may be modulatory rather than activating.
An important feature of the current study was our ability to examine the effects of reactivating the network. We observed that reactivation of a movement domain in $\mathrm{M} 1$ resulted in a return to the baseline evoked movement in PPCr at 12 of 19 sites and increases or decreases in the amplitude of the movement occurred at four of 19 sites. There are several explanations of why reactivation of M1 did not result in a return to baseline movements in PPCr at all sites tested. The first is that this result is a technical artifact, possibly due to changes in excitability over time with repeated stimulation. We do not think that this is the case because, in similar studies in our laboratory in macaque monkeys, during sham cooling, in which an identical protocol was followed without the actual cooling, there was no change in the amplitude or type of evoked movements (Cooke et al., 2015). A second explanation is that cooling (or rewarming) itself contributed to persistent changes. Our study did not distinguish the effects of deactivation from those of the cooling that caused it. Therefore, it is possible that the persistent effects that we observed were caused (in part or whole) more directly by a change in temperature. For example, studies of slice preparations have reported supernormal neural excitability during rewarming (Aihara et al., 2001; Volgushev et al., 2004). This by itself would seem likely to end rather than continue effects of cooling, but could also contribute to longer-term changes. A third explanation is that deactivation via cooling resulted in rapid plasticity, which reweighted the network. This is supported by previous work by us and others demonstrating that cooling deactivation and subsequent reactivation of cortical networks do not always result in a return to baseline in neural response properties (Clemo and Stein, 1986; Carrasco and Lomber, 2009; Girardin and Martin, 2009; Goldring et al., 2014). Previously, we proposed that recent changes in the activity of the circuit altered synaptic weights, that the neocortex is primed for future processing based on an immediate peristimulus event, and that sensorimotor networks are highly dynamic and change from minute to minute depending on the overall activity of the network (Goldring et al., 2014). This appears to be true for frontoparietal networks as well. As noted earlier, $\mathrm{PPCr}$ processes multiple sensory and motor inputs and has been proposed to be involved in the selection of appropriate movement domains and in the inhibition of others. Selection of combinations of cortical motor primitives (movement domains) could yield a large repertoire of behavioral outcomes (Mussa-Ivaldi and Bizzi, 2000). However, this "decision making" network is not ballistic, but must be continually updated based on the immediate context or state of the network (Cisek and Kalaska, 2010) and on inputs from motor and somatosensory cortex supplying information on the current configuration of the body or "feedback remapping" (Graziano, 2006). These ideas are compatible with previous work in macaque monkeys indicating that decisions emerge from competing options and that the network requires continuous updating of relevant sensory and proprioceptive systems, as well as internal factors such as the goal value and the cost of movement (Christopoulos et al., 2015).

\section{References}

Aihara H, Okada Y, Tamaki N (2001) The effects of cooling and rewarming on the neuronal activity of pyramidal neurons in guinea pig hippocampal slices. Brain Res 893:36-45. CrossRef Medline

Andersen RA, Andersen KN, Hwang EJ, Hauschild M (2014) Optic ataxia: from Balint's syndrome to the parietal reach region. Neuron 81:967-983. CrossRef Medline 
Asanuma H, Rosén I (1972) Topographical organization of cortical efferent zones projecting to distal forelimb muscles in the monkey. Exp Brain Res 14:243-256. CrossRef Medline

Bakola S, Passarelli L, Gamberini M, Fattori P, Galletti C (2013) Cortical connectivity suggests a role in limb coordination for macaque area PE of the superior parietal cortex. J Neurosci 33:6648-6658. CrossRef Medline

Baldwin MK, Cooke DF, Gordon A, Krubitzer L (2014) Revealing functional organization of frontoparietal networks in tree shrews (Tupaia belangeri) using reversible inactivation. Soc Neurosci Abstr 40:446.02.

Brown AR, Teskey GC (2014) Motor cortex is functionally organized as a set of spatially distinct representations for complex movements. J Neurosci 34:13574-13585. CrossRef Medline

Burman KJ, Palmer SM, Gamberini M, Spitzer MW, Rosa MG (2008) Anatomical and physiological definition of the motor cortex of the marmoset monkey. J Comp Neurol 506:860-876. CrossRef Medline

Carrasco A, Lomber SG (2009) Evidence for hierarchical processing in cat auditory cortex: nonreciprocal influence of primary auditory cortex on the posterior auditory field. J Neurosci 29:14323-14333. CrossRef Medline

Christopoulos V, Bonaiuto J, Andersen RA (2015) A biologically plausible computational theory for value integration and action selection in decisions with competing alternatives. PLoS Comput Biol 11:e1004104. CrossRef Medline

Cisek P, Kalaska JF (2010) Neural mechanisms for interacting with a world full of action choices. Annu Rev Neurosci 33:269-298. CrossRef Medline

Clemo HR, Stein BE (1986) Effects of cooling somatosensory cortex on response properties of tactile cells in the superior colliculus. J Neurophysiol 55:1352-1368. Medline

Colby CL (1998) Action-oriented spatial reference frames in cortex. Neuron 20:15-24. CrossRef Medline

Cooke DF, Graziano MS (2004) Sensorimotor integration in the precentral gyrus: polysensory neurons and defensive movements. J Neurophysiol 91:1648-1660. CrossRef Medline

Cooke DF, Taylor CS, Moore T, Graziano MS (2003) Complex movements evoked by microstimulation of the ventral intraparietal area. Proc Natl Acad Sci U S A 100:6163-6168. CrossRef Medline

Cooke DF, Padberg J, Zahner T, Krubitzer L (2012a) The functional organization and cortical connections of motor cortex in squirrels. Cereb Cortex 22:1959-1978. CrossRef Medline

Cooke DF, Goldring AB, Yamayoshi I, Tsourkas P, Recanzone GH, Tiriac A, Pan T, Simon SI, Krubitzer L (2012b) Fabrication of an inexpensive, implantable cooling device for reversible brain deactivation in animals ranging from rodents to primates. J Neurophysiol 107:3543-3558. CrossRef Medline

Cooke DF, Goldring AB, Baldwin MK, Recanzone GH, Chen A, Pan T, Simon SI, Krubitzer L (2014) Reversible deactivation of higher-order posterior parietal areas. I. Alterations of receptive field characteristics in early stages of neocortical processing. J Neurophysiol 112:2529-2544. CrossRef Medline

Cooke DF, Goldring AB, Baldwin MKL, Donaldson MS, Krubitzer L (2015) Reversible deactivation of motor cortex reveals functional connectivity with anterior and posterior parietal cortex in Old World monkeys (Macaca mulatta). Neurosci Abs.

Culham JC, Cavina-Pratesi C, Singhal A (2006) The role of parietal cortex in visuomotor control: what have we learned from neuroimaging? Neuropsychologia 44:2668-2684. CrossRef Medline

Flash T, Hochner B (2005) Motor primitives in vertebrates and invertebrates. Curr Opin Neurobiol 15:660-666. CrossRef Medline

Fogassi L, Gentilucci M, Luppino G, Matelli M, Rizzolatti G (1986) The motor cortex of Galago crassicaudatus: a morphological and electrophysiological study [article in Italian]. Boll Soc Ital Biol Sper 62:1067-1072. Medline

Fogassi L, Gallese V, Gentilucci M, Luppino G, Matelli M, Rizzolatti G (1994) The fronto-parietal cortex of the prosimian Galago: patterns of cytochrome oxidase activity and motor maps. Behav Brain Res 60:91-113. CrossRef Medline

Galea MP, Darian-Smith I (1994) Multiple corticospinal neuron populations in the macaque monkey are specified by their unique cortical origins, spinal terminations, and connections. Cereb Cortex 4:166-194. CrossRef Medline

Gallyas F (1979) Silver staining of myelin by means of physical development. Neurology 1:203-209. Medline
Gharbawie OA, Stepniewska I, Kaas JH (2011a) Cortical connections of functional zones in posterior parietal cortex and frontal cortex motor regions in new world monkeys. Cereb Cortex 21:1981-2002. CrossRef Medline

Gharbawie OA, Stepniewska I, Qi H, Kaas JH (2011b) Multiple parietalfrontal pathways mediate grasping in macaque monkeys. J Neurosci 31: 11660-11677. CrossRef Medline

Ghosh S, Brinkman C, Porter R (1987) A quantitative study of the distribution of neurons projecting to the precentral motor cortex in the monkey (M. fascicularis). J Comp Neurol 259:424-444. CrossRef Medline

Girardin CC, Martin KA (2009) Cooling in cat visual cortex: stability of orientation selectivity despite changes in responsiveness and spike width. Neuroscience 164:777-787. CrossRef Medline

Goldring AB, Cooke DF, Baldwin MK, Recanzone GH, Gordon AG, Pan T, Simon SI, Krubitzer L (2014) Reversible deactivation of higher-order posterior parietal areas. II. Alterations in response properties of neurons in areas 1 and 2. J Neurophysiol 112:2545-2560. CrossRef Medline

Goode GE, Haines DE (1975) Origin, course and termination of corticospinal fibers in a prosimian primate, Galago. Brain Behav Evol 12:334-360. CrossRef Medline

Gould HJ 3rd, Cusick CG, Pons TP, Kaas JH (1986) The relationship of corpus callosum connections to electrical stimulation maps of motor, supplementary motor, and the frontal eye fields in owl monkeys. J Comp Neurol 247:297-325. CrossRef Medline

Graziano M (2006) The organization of behavioral repertoire in motor cortex. Annu Rev Neurosci 29:105-134. CrossRef Medline

Graziano MS, Taylor CS, Moore T, Cooke DF (2002) The cortical control of movement revisited. Neuron 36:349-362. CrossRef Medline

Graziano MS, Aflalo TN, Cooke DF (2005) Arm movements evoked by electrical stimulation in the motor cortex of monkeys. J Neurophysiol 94: 4209-4223. CrossRef Medline

Hikosaka O, Wurtz RH (1985) Modification of saccadic eye movements by GABA-related substances. I. Effect of muscimol and bicuculline in monkey superior colliculus. J Neurophysiol 53:266-291. Medline

Hinkley LB, Krubitzer LA, Padberg J, Disbrow EA (2009) Visual-manual exploration and posterior parietal cortex in humans. J Neurophysiol 102: 3433-3446. CrossRef Medline

Hwang EJ, Hauschild M, Wilke M, Andersen RA (2014) Spatial and temporal eye-hand coordination relies on the parietal reach region. J Neurosci 34:12884-12892. CrossRef Medline

Jones EG, Coulter JD, Hendry SH (1978) Intracortical connectivity of architectonic fields in the somatic sensory, motor and parietal cortex of monkeys. J Comp Neurol 181:291-347. CrossRef Medline

Kaas JH, Stepniewska I (2015) The evolution of posterior parietal cortex and parietal-frontal networks for specific actions in primates. J Comp Neurol. In press.

Kaas JH, Gharbawie OA, Stepniewska I (2011) The organization and evolution of dorsal stream multisensory motor pathways in primates. Front Neuroanat 5:34. Medline

Kaas JH, Gharbawie OA, Stepniewska I (2013) Cortical networks for ethologically relevant behaviors in primates. Am J Primatol 75:407-414. CrossRef Medline

Lewis JW, Van Essen DC (2000) Corticocortical connections of visual sensorimotor, multimodal processing areas in the parietal lobe of the macaque monkey. J Comp Neurol 428:112-137. CrossRef Medline

Malpeli JG (1999) Reversible inactivation of subcortical sites by drug injection. J Neurosci Methods 86:119-128. CrossRef Medline

Maranesi M, Rodà F, Bonini L, Rozzi S, Ferrari PF, Fogassi L, Coudé G (2012) Anatomo-functional organization of the ventral primary motor and premotor cortex in the macaque monkey. Eur J Neurosci 36:3376-3387. CrossRef Medline

Martin JH (1991) Autoradiographic estimation of the extent of reversible inactivation produced by microinjection of lidocaine and muscimol in the rat. Neurosci Lett 127:160-164. CrossRef Medline

Martin JH, Ghez C (1993) Differential impairments in reaching and grasping produced by local inactivation within the forelimb representation of the motor cortex in the cat. Exp Brain Res 94:429-443. Medline

McGuinness E, Sivertsen D, Allman JM (1980) Organization of the face representation in macaque motor cortex. J Comp Neurol 193:591-608. CrossRef Medline

Murray EA, Coulter JD (1981) Organization of corticospinal neurons in the monkey. J Comp Neurol 195:339-365. CrossRef Medline 
Mussa-Ivaldi FA, Bizzi E (2000) Motor learning through the combination of primitives. Philos Trans R Soc Lond B Biol Sci 355:1755-1769. CrossRef Medline

Nudo RJ, Masterton RB (1990) Descending pathways to the spinal cord, III: Sites of origin of the corticospinal tract. J Comp Neurol 296:559-583. CrossRef Medline

Padberg J, Recanzone G, Engle J, Cooke D, Goldring A, Krubitzer L (2010) Lesions in posterior parietal area 5 in monkeys result in rapid behavioral and cortical plasticity. J Neurosci 30:12918-12935. CrossRef Medline

Ponce CR, Hunter JN, Pack CC, Lomber SG, Born RT (2011) Contributions of indirect pathways to visual response properties in macaque middle temporal area MT. J Neurosci 31:3894-3903. CrossRef Medline

Remple MS, Reed JL, Stepniewska I, Lyon DC, Kaas JH (2007) The organization of frontoparietal cortex in the tree shrew (Tupaia belangeri): II. Connectional evidence for a frontal-posterior parietal network. J Comp Neurol 501:121-149. CrossRef Medline

Rozzi S, Ferrari PF, Bonini L, Rizzolatti G, Fogassi L (2008) Functional organization of inferior parietal lobule convexity in the macaque monkey: electrophysiological characterization of motor, sensory and mirror responses and their correlation with cytoarchitectonic areas. Eur J Neurosci 28:1569-1588. CrossRef Medline

Stepniewska I, Preuss TM, Kaas JH (1993) Architectonics, somatotopic organization, and ipsilateral cortical connections of the primary motor area (MI) of owl monkeys. J Comp Neurol 330:238-271. CrossRef Medline

Stepniewska I, Fang PC, Kaas JH (2005) Microstimulation reveals specialized subregions for different complex movements in posterior parietal cortex of prosimian galagos. Proc Natl Acad Sci U S A 102:4878-4883. CrossRef Medline

Stepniewska I, Fang PC, Kaas JH (2009a) Organization of the posterior parietal cortex in galagos: I. Functional zones identified by microstimulation. J Comp Neurol 517:765-782. CrossRef Medline
Stepniewska I, Cerkevich CM, Fang PC, Kaas JH (2009b) Organization of the posterior parietal cortex in galagos: II. Ipsilateral cortical connections of physiologically identified zones within anterior sensorimotor region. J Comp Neurol 517:783-807. CrossRef Medline

Stepniewska I, Friedman RM, Gharbawie OA, Cerkevich CM, Roe AW, Kaas JH (2011) Optical imaging in galagos reveals parietal-frontal circuits underlying motor behavior. Proc Natl Acad Sci U S A 108:E725-E732. CrossRef Medline

Stepniewska I, Gharbawie OA, Burish MJ, Kaas JH (2014) Effects of muscimol inactivations of functional domains in motor, premotor, and posterior parietal cortex on complex movements evoked by electrical stimulation. J Neurophysiol 111:1100-1119. CrossRef Medline

Strick PL, Kim CC (1978) Input to primate motor cortex from posterior parietal cortex (area 5). I. Demonstration by retrograde transport. Brain Res 157:325-330. CrossRef Medline

Thier P, Andersen RA (1996) Electrical microstimulation suggests two different forms of representation of head-centered space in the intraparietal sulcus of rhesus monkeys. Proc Natl Acad Sci U S A 93:4962-4967. CrossRef Medline

Volgushev M, Kudryashov I, Chistiakova M, Mukovski M, Niesmann J, Eysel UT (2004) Probability of transmitter release at neocortical synapses at different temperatures. J Neurophysiol 92:212-220. CrossRef Medline

Wong P, Kaas JH (2010) Architectonic subdivisions of neocortex in the Galago (Otolemur garnetti). Anat Rec (Hoboken) 293:1033-1069. CrossRef Medline

Wong-Riley M (1979) Changes in the visual system of monocularly sutured or enucleated cats demonstrable with cytochrome oxidase histochemistry. Brain Res 171:11-28. CrossRef Medline

Wu CW, Bichot NP, Kaas JH (2000) Converging evidence from microstimulation, architecture, and connections for multiple motor areas in the frontal and cingulate cortex of prosimian primates. J Comp Neurol 423: 140-177. CrossRef Medline 